\title{
Rationalizing the lacking of inversion symmetry in a non-centrosymmetric polar racemate: an experimental and theoretical study
}

$\underline{\text { Leonardo Lo Presti }}^{*, \forall, \#,+}$ Mattia Sist, ${ }^{\#}$ Laura Loconte, ${ }^{\forall}$ Andrea Pinto, ${ }^{\partial}$ Lucia Tamborini ${ }^{\partial}$ and Carlo Gatti $^{\#,+}$

${ }^{\forall}$ Università degli Studi di Milano, Department of Chemistry, Via Golgi 19 I-20133 Milano (Italy)

\# Center for Materials Crystallography, Aarhus University, Langelandsgade 140, 8000 Aarhus, Denmark

+ CNR-ISTM, Istituto di Scienze e Tecnologie Molecolari,, Via Golgi 19 I-20133 Milano (Italy)

ว Dipartimento di Scienze Farmaceutiche, Università degli Studi di Milano,Via Mangiagalli 25, 20133 Milano, Italy

* To whom correspondence should be addressed: leonardo.lopresti@unimi.it

\section{PLEASE CITE THIS WORK AS}

L. Lo Presti, M. Sist, L. Loconte. A. Pinto, L. Tamborini, C. Gatti, Crystal Growth \& Design 2014, 14, 5822-5833 DOI: http://pubs.acs.org/doi/abs/10.1021/cg501074x 


\begin{abstract}
The total charge density of PYRAC, a polar $\left(P c a 2_{1}\right)$ organic racemate with $Z^{\prime}=2$ was derived from high-resolution single-crystal X-ray diffraction data at $T=100(2) \mathrm{K}$ and periodic DFT calculations. The PYRAC asymmetric unit consists of a hydrogen-bonded pair of conformationally different enantiomers, $\mathrm{A}$ and $\mathrm{B}_{\mathrm{i}}$, where the subscript ' $\mathrm{i}$ ' indicates a reversed absolute configuration. The lattice stability was compared with that of centrosymmetric possibly competing structures, with the aim of understanding why a non-centrosymmetric lattice framework is obtained from a racemic mixture. The likelihood of specific intermolecular recognition processes among different conformers of PYRAC in the very first stages of nucleation was investigated by DFT simulations in vасио. Two competing, equivalent interconversion pseudorotatory paths between the most stable A and the least stable B conformers were found. It results that molecules spend most of their time $(\approx$ $53 \%)$ in the $\mathrm{A}$ conformation, while the $\mathrm{B}$ one is far less populated $(\approx 7 \%)$. Therefore, centrosymmetric $\mathrm{AA}_{\mathrm{i}}$ adducts are formed very frequently in the reaction liquor, while the $\mathrm{BB}_{\mathrm{i}}$ ones are rare. Nevertheless, $\mathrm{AA}_{\mathrm{i}}$ pairs produce crystal forms with cohesive energies and densities significantly less favourable than those estimated for the non-centrosymmetric heterochiral $A_{i}$ ones. Therefore, preference for $Z=2$ in conjunction with non-centrosymmetric point and space groups results from the thermodynamic control of the crystallization process. The capability of forming extended hydrogen bond chains throughout the lattice appears to be a prerequisite to bind together the fundamental $\mathrm{AB}_{\mathrm{i}}$ repeating units.
\end{abstract}

Keywords: Single-crystal X-ray diffraction, kinetics of conformational changes, racemate, polar space groups, cohesive energy 


\section{Introduction}

It is well known that several bulk properties of crystalline compounds rely on symmetry at atomic, molecular and supramolecular levels. Symmetry is perhaps the most fundamental property of matter, ${ }^{1}$ as it sets up exact requirements that any observable quantity must fulfil. ${ }^{2}$ Several physical effects that raise interest in cutting-edge research fields, such as the design of smart materials and advanced sensors, depend on the (strong) anisotropic coupling among crystal lattice, electron polarizability and an external field producing mechanical, thermal or electromagnetic stress. 'Anisotropic' is the key term in this context: for example piezoelectricity, ${ }^{3}$ pyroelectricity ${ }^{4}$ and second-harmonic generation ${ }^{5}$ cannot manifest in substances with centrosymmetric crystallographic point groups, as in these latter any externally triggered mechnical or electric distortion would be counteracted by an equal and opposite structural or electronic response. Therefore, it is not surprising that lacking of inversion symmetry is the first and most striking requirement sought for in designing several types of advanced materials, although symmetry axes and planes may also play a decisive role. ${ }^{2}$ For example, a unique axis compatible with a nonvanishing first momentum of the charge density distribution in the whole unit cell is mandatory for pyroelectricity. An acentric space group where such an axis exists is called 'polar'. For a crystal structure to be truly polar, however, a non-vanishing electric dipole moment directed along that axis is also required. ${ }^{6}$

Predicting and hopefully controlling crystal symmetry in dependence of specific synthetic conditions is one of the main tasks of modern direct ${ }^{7}$ and reverse ${ }^{8}$ crystal engineering. A great deal of effort has been spent in the last decade on this topic, ${ }^{9}$ with major focus on molecular crystals. ${ }^{10}$ Nevertheless, it is still virtually impossible to know in advance which space group, or even which crystal system a new molecular compound will exploit in the solid state. ${ }^{10 c}$ The problem is discouragingly intricate, as crystal nucleation and growth kinetics are as important as thermodynamics in governing the crystallization process, ${ }^{11}$ and there is no way to know from the molecular structure alone whether a kinetic polymorph will be also thermodynamically favoured or not. ${ }^{12}$ A further level of complexity arises when conformational differences occur in the solid with respect to isolated molecules. Crystal structures of flexible molecules are still not predictable from scratch, even though in the most recent 'blind test' ${ }^{13}$ two groups out of a total of 14 found the correct structure of a medium-size benzylcarbamate derivative with high torsional freedom. The successful predictions yet made still use of database-stored information. In this respect, single-crystal X-ray diffraction analysis of complex systems represents a valuable and complementary approach to computer simulations ${ }^{14}$ if specimens of sufficient quality are available. ${ }^{15}$ 


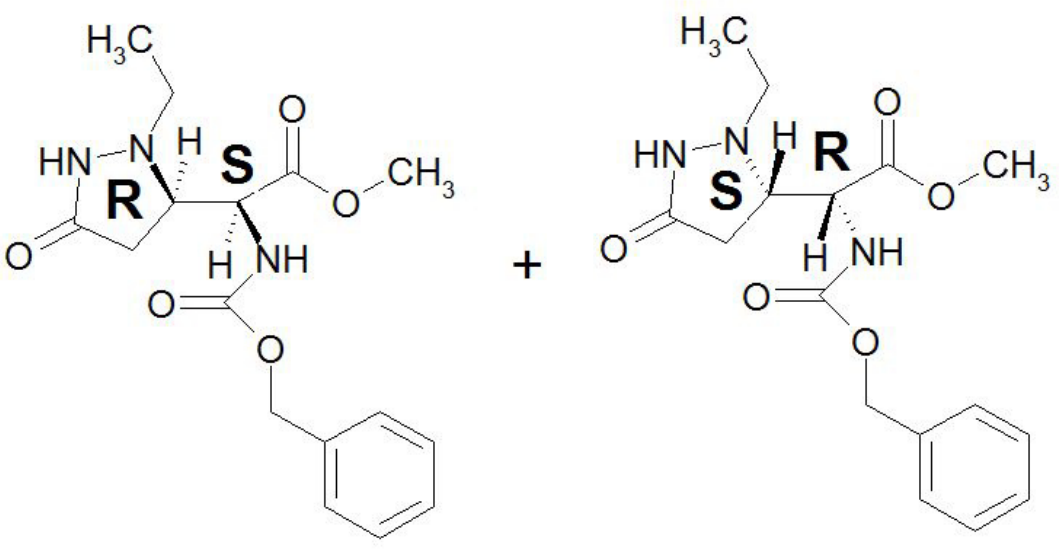

Scheme 1

In this work, we report on the experimental and theoretical study of a racemic aminoacid pyrazolidine derivative (hereinafter PYRAC, $\mathrm{C}_{16} \mathrm{H}_{21} \mathrm{~N}_{3} \mathrm{O}_{5}$ ), 3-pyrazolidineacetic acid, 2-ethyl-5oxo- $\alpha-[[($ phenylmethoxy)carbonyl]amino]- methyl ester, $(\alpha \mathrm{R}, 3 \mathrm{~S})-\mathrm{rel}$, a synthesis intermediate of promising cytidine triphosphate (CTP) synthetase inhibitors against the African Trypanosomiasis. ${ }^{16}$ PYRAC (Scheme 1) crystallizes in the acentric polar space group Pca2 ${ }_{1}\left(\mathrm{n}^{\circ} 29\right)$ with 8 formulae in cell. In fact, its asymmetric unit contains a couple of hydrogen-bonded enantiomers in distinct conformations. ${ }^{16}$ This is not surprising per se, as non-centrosymmetric racemates are not rare. Out of a sample of 5560 unique centrosymmetric organic structures stored in the Cambridge Structural Database (CSD), ${ }^{17,18}$ Dalhus \& Görbitz estimate ${ }^{19}$ that $23 \%$ are racemates. Their occurrence falls to $16.5 \%$ in a sample of 3819 mirror-symmetric acentric space groups. Conversely, chiral space groups are hardly preferred in racemates, and - apart very few exceptions ${ }^{19,20}$ - one anticipates inversion and/or reflection elements to be present for pairs of opposite enantiomers. However, the question on what factors influence the probability of inversion symmetry to occur remains open. When spontaneous resolution ${ }^{21}$ does not take place, there are no obvious explanations of why a noncentrosymmetric or even a chiral lattice framework comes out from a racemic mixture. The reason may be thermodynamic, or kinetic, or both, and ultimately depends on how individual conformers recognize each other in the very first stages of the nucleation process. In this context, joint theoretical first-principle and experimental studies are becoming increasingly popular ${ }^{22}$ for accurate intermolecular (self)-recognition studies in the solid state.

Our present work is aimed at providing a rationale for the relative stability of the observed crystal phase with respect to stable and close-packed centric competing structures, so to get a model able to justify the lack of inversion symmetry in PYRAC. To this end, from accurate single-crystal X-ray diffraction experiments at $T=100 \mathrm{~K}$, we derived the total charge density of PYRAC and its crystal cohesive energy. Experimental outcomes were complemented by DFT simulations in vacuo and in the bulk, as well as by Hirshfeld surface analysis and force-field based calculations. PYRAC 
is also a good test case to look at how high conformational flexibility can result in different accessible classes of intermolecular interaction patterns. We thus explored the kinetics of the pseudorotatory path among the experimentally detected conformations by DFT simulations on the in vacuo Born-Oppenheimer surface. The focus here is on the thermodynamics and kinetics of the isolated system, upon which the self-recognition mechanism of PYRAC must rely. Inclusion of solvent effects is delayed to forthcoming studies.



Figure 1. Colour online. Asymmetric unit (ASU) of PYRAC at $T=100(2) \mathrm{K}$, with the atom numbering scheme. Thermal ellipsoids are drawn at the $50 \%$ probability level and intermolecular cyclic $\mathrm{NH} \cdots \mathrm{O}$ hydrogen bonds are highlighted as purple dotted lines. In this picture, $\mathrm{A}$ and $\mathrm{B}$ correspond to $\mathrm{C} 3 \mathrm{~A}(\mathrm{R}), \mathrm{C} 6 \mathrm{~A}(\mathrm{~S})$ and $\mathrm{C} 3 \mathrm{~B}(\mathrm{~S}), \mathrm{C} 6 \mathrm{~B}(\mathrm{R})$ absolute configurations. Inset: crystal employed in the present diffraction analysis. The sample is $\approx 550 \mu \mathrm{m}$ long. All the molecular pictures in this work were realized with Diamond v.3.2i, K. Brandenburg, (C) 1997-2012 Crystal Impact GbR, Bonn, Germany.

\section{Materials and Methods.}

2.1 X-ray diffraction and experimental charge density. PYRAC (Figure 1) was synthesized as described elsewhere. ${ }^{16}$ Large and well-formed crystals appeared directly after resting the reaction liquor overnight at RT from a 1:1 mixture of ethyl acetate/hexane. ${ }^{23}$ The pure compound was kept in the dark in a refrigerator at $T=4{ }^{\circ} \mathrm{C}$ for $\approx 2.5$ months. Nine specimens, sampled from different zones of the glass tube (including its walls), were tested at the diffractometer until a charge-density grade $^{15}$ crystal was found. Neither other crystal forms were found in the original batch of material by powder diffraction methods (Section S1 of the Supporting Information, SI), nor solid-solid phase 
transitions were detected in the whole $T$ range explored up to the melting point $\left(\approx 92{ }^{\circ} \mathrm{C}\right)$. Slow cooling $\left(1^{\circ} \mathrm{C} / \mathrm{min}\right)$ of the melt resulted in a glassy state (see Section $\left.\mathrm{S} 4, \mathrm{SI}\right)$.

Table 1. Crystallographic details and refinement statistics of PYRAC $\left(\mathrm{C}_{16} \mathrm{H}_{21} \mathrm{~N}_{3} \mathrm{O}_{5}\right.$, orthorhombic, $\left.P c a 2_{1}, F_{000}=1424 e, M_{w}=335.36 \mathrm{amu}\right)$.



${ }^{\text {a }} Z$ is the number of formulae per cell, whereas $Z$ ' is the number of formulae in the ASU

The ASU consists of a pair of conformationally different enantiomers, hereinafter labelled as ' $A$ ' and ' $B_{i}$ ' (see below), with the ' $\mathrm{i}$ ' subscript denoting their opposite chirality. X-ray diffraction data were collected at $T=100(2) \mathrm{K}$ using graphite-monochromated Mo $\mathrm{K} \alpha$ radiation $(\lambda=0.71073$ $\AA$ ) at a nominal source power of $50 \mathrm{kV}$ x $30 \mathrm{~mA}$ on a three-circle Bruker SMART APEX II goniometer equipped with a CCD area detector and an Oxford Cryosystems $\mathrm{N}_{2}$ gas blower. The $\mathrm{SAINT}^{24}$ program package was employed to perform integration and preliminary data reduction, whereas beam anisotropy corrections and final scaling were applied by SADABS ${ }^{25}$ and XPREP. ${ }^{26}$ The final dataset was $\approx 99 \%$ complete up to the maximum resolution of $1.0 \AA^{-1}$ in $\sin \vartheta / \lambda$ (Table 1 ). The static experimental charge density $\left(\rho_{\text {EXP }}\right)$ was extracted from the observed structure factor squared amplitudes $\left(F_{\exp }{ }^{2}\right)$ at $T=100 \mathrm{~K}$ through the Hansen-Coppens multipole formalism ${ }^{27}$ as implemented in the XD2006 software package. ${ }^{28}$ Relevant refinement details and agreement statistics can be found in Table 1, while a detailed discussion on the least-squares strategy and on the treatment of the thermal motion can be found in Sections S2 and S4 SI. The Quantum Theory of Atoms in Molecules ${ }^{29 a}$ was employed throughout to derive topological descriptors ${ }^{29 b, c}$ from the $\rho_{\text {EXP }}$ distribution. 
2.2 Bulk DFT simulations. The LCGTF (Linear Combination of Gaussian-Type Functions) approach as implemented in the CRYSTAL09 $\operatorname{code}^{30 \mathrm{a}}$ was employed to perform all-electron quantum-mechanical simulations in bulk PYRAC. A double-zeta 6-31G(p,d) basis set with exponents suitably modified for solid-state calculations ${ }^{31}$ was selected in conjunction with the hybrid B3LYP functional. ${ }^{32}{ }^{3030}$ Unique theoretical structure factor amplitudes with the same indices as the measured data were also computed. They were employed to derive a multipole-projected electron density distribution, $\rho_{\text {THEO }}$, fully consistent with the $\rho_{\text {EXP }}$ one in terms of considered structure factors and multipole model expansion. Full technical details are reported in Section S3, SI.

2.3 Search for possible competing polymorphic centrosymmetric structures. The CoulombLondon-Pauli (CLP) model of intermolecular interactions as implemented in the CLP program package $^{33}$ was employed to generate possible PYRAC polymorphs based on the fully centrosymmetric $\mathrm{XX}_{\mathrm{i}}$ molecular pairs, with $\mathrm{X}$ denoting one of the stable conformers found in the gas-phase (see Sections 3.2.3 below and S8, SI) and $\mathrm{X}_{\mathrm{i}}$ its corresponding inversion-related analogue. First, the $\mathrm{XX}_{\mathrm{i}}$ geometries were optimized in vacuo at the DFT B3LYP ${ }^{32} 6-311 \mathrm{G}(\mathrm{p}, \mathrm{d})^{34}$ theory level by means of the Gaussian09 program. ${ }^{35}$ Then, the Clpoly module of the CLP package built a set of possible centrosymmetric crystal structures with one PYRAC molecule in the ASU. The current version of the program can handle a limited number of space groups, so we focused on $P \overline{1}, P 2{ }_{1} / c$, $C 2 / c$ and Pbca lattice symmetries that account for roughly the $90 \%$ of the centrosymmetric structures in the up-to-date version of the CSD. ${ }^{18}$ To compare the competing possible polymorphs of PYRAC on the same grounds, the experimental structure and the most stable CLP-derived structures were employed as starting guesses to perform all-electron periodic DFT optimizations by means of the CRSYTAL09 program $^{30 b, c}$ at the B3LYP 6-31G(p,d ${ }^{31}$ level of theory (see Section S3, SI).

2.4 In vacuo simulations. Quantum-mechanical simulations were performed on isolated molecules and suitable adducts at the $6-311 \mathrm{G}(\mathrm{p}, \mathrm{d})^{34}$ level of theory in conjunction with the B3LYP ${ }^{32}$ DFT functional, through the Gaussian09 program package. ${ }^{35}$ Full structure optimizations were performed on both isolated conformers and interacting centrosymmetric $\mathrm{XX}_{\mathrm{i}}$ pairs (see above). All the molecule-molecule interaction energies were corrected for zero-point energy (ZPE) and basis set superposition error (BSSE). ${ }^{36} \mathrm{~A}$ possible interconversion mechanism between the $\mathrm{A}$ and $\mathrm{B}$ arrangements of the $\mathrm{C} 3(\mathrm{~S}), \mathrm{C} 6(\mathrm{R})$ enantiomer was determined by a transition-state optimization 
procedure in vacuo (Section 3.2.2). Full details are reported in the Supporting Information (Section S6, Figures S8-S12).

\section{Results and discussion.}

The paper is organized as follows. First, the experimental packing of PYRAC is described (Section 3.1). Then, a plausible mechanism for the molecular self-recognition in vacuo is elucidated (Section 3.2) and possibly competing centrosymmetric polymorphs of PYRAC are looked for through first-principle simulations (Section 3.3). Conclusions are drawn in Section 4.

\subsection{Crystal packing in PYRAC: analysis of the experimental structure}

3.1.1 Hydrogen bond patterns. PYRAC lacks hydroxyl functions; just two possible $\mathrm{N}-\mathrm{H}$ hydrogen bond (HB) donors are available.

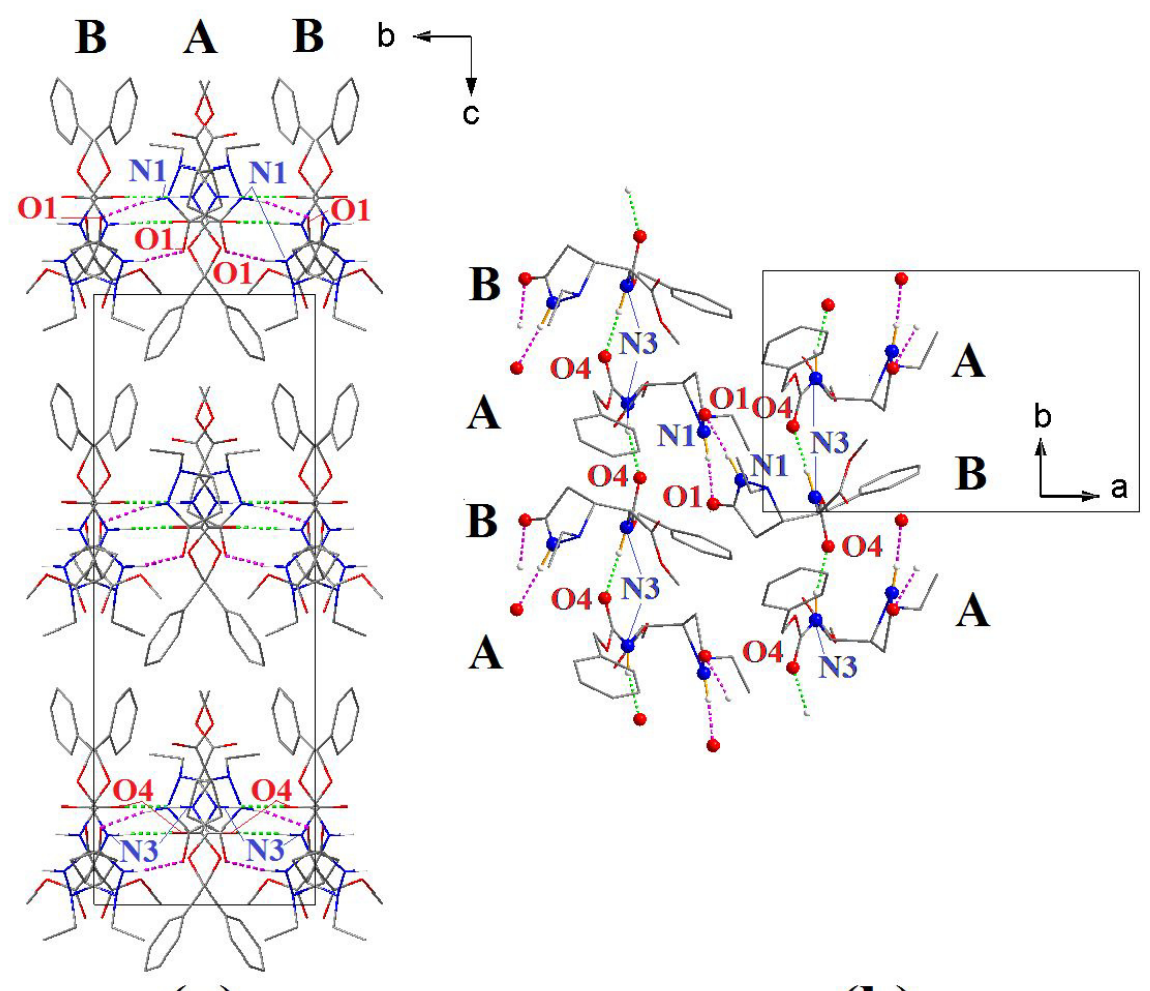

(a)

(b)

Figure 2. Colour online. Wires-and-stick representation of the crystal packing of PYRAC at $T=$ $100(2) \mathrm{K}$, with alternating layers of A and B conformers along $b$ highlighted (see text). Hydrogen atoms not involved in the specific HBs discussed in the text have been omitted for clarity.

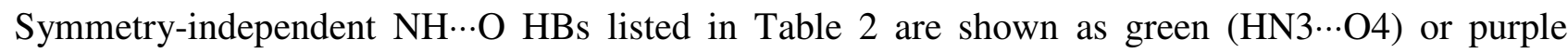

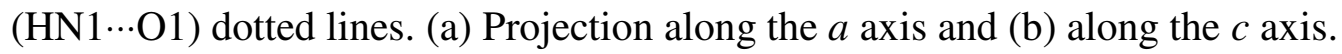

The latter belong to the carbammate $(\mathrm{N} 3-\mathrm{H})$ and the pyrazolidinone $(\mathrm{N} 1-\mathrm{H})$ systems (Figure 1, Scheme 1) and are all saturated in the $\operatorname{crystal}^{37}$ (Figure 2, Table 2). Different A and B conformers are piled up along the $b$ axis, forming alternating layers of segregated A or B molecules almost 
parallel to the $(a, c)$ plane (Figure 2). Adjacent planes along $b$ are held together through two independent $\mathrm{N} 3-\mathrm{H} \cdots \mathrm{O} 4$ hydrogen bonds, that form infinite zig-zag chains of alternating -A-B-A-Bconformers (green dotted lines in Figure 2). The latter are enantiomerically related to each other through the $a$ glide plane (Table 2). The N1-H $\cdots \mathrm{O} 1 \mathrm{HBs}$, on the other hand, set up a cyclic pattern between facing pyrazolidinone rings of independent molecules in the ASU (purple dotted lines in Figures 1 and 2), and bridge parallel -A-B-A-B- chains along the a direction (Figure 2b). Much weaker $\mathrm{CH} \cdots \mathrm{O}$ interactions (Table S3, SI) are set up among molecules lying in planes orthogonal to b. The anisotropy in directional HB interactions governs the cell edge changes when the temperature is lowered, as the $b$ axis undergoes a significantly smaller relative contraction $(-0.99(1)$ $\%)$ with respect to the $a(-1.89(1) \%)$ and $c(-1.54(1) \%)$ axes upon cooling from RT to $100 \mathrm{~K}$. Not unexpectedly, the unit cell preferentially shrinks along directions exploiting less tight binding. ${ }^{38}$

Table 2. Geometrical and point topological descriptors obtained from the experimental multipole model, $\rho_{\text {EXP }}$, of the $\mathrm{NH} \cdots \mathrm{O}$ hydrogen-bonded contacts shown in Figures 1 and 2. When available, estimated standard deviations are reported in parentheses.

\begin{tabular}{|c|c|c|c|c|c|c|c|}
\hline $\mathrm{N}-\mathrm{H} \cdots \mathrm{O}$ & $d_{\mathrm{N}-\mathrm{H}} / \AA^{\mathrm{a}}$ & $d_{\mathrm{N} \cdots \mathrm{O}} / \AA$ & $d_{\mathrm{H} \cdots \mathrm{O}} / \AA$ & $\alpha_{\mathrm{NHO}} / \mathrm{deg}$ & $\rho_{\mathrm{bcp}} \mathrm{b} / \mathrm{e} \cdot \AA^{-3}$ & $\nabla^{2} \rho_{\text {bcp }} \mathrm{b} / \mathrm{e} \cdot \AA^{-5}$ & symmetry $^{\mathrm{c}}$ \\
\hline N1A-HN1A $\cdots$ O1B & 1.027 & $2.9113(8)$ & 1.90 & 168.6 & $0.17(5)$ & $1.6(1)$ & $\mathrm{x}, \mathrm{y}, \mathrm{z}$ \\
\hline N1B-HN1B $\cdots$ O1A & 1.027 & $2.8969(8)$ & 1.89 & 170.9 & $0.18(5)$ & $1.97(9)$ & $x, y, z$ \\
\hline  & 1.027 & $2.8525(7)$ & 1.84 & 167.6 & $0.13(6)$ & $2.8(1)$ & $\mathrm{x}-1 / 2,-\mathrm{y}, \mathrm{z}$ \\
\hline N3B-HN3B $\cdots \mathrm{O} 4 \mathrm{~A}$ & 1.027 & $2.8415(7)$ & 1.82 & 177.1 & $0.27(4)$ & $0.98(6)$ & $x+1 / 2,-y+1, z$ \\
\hline
\end{tabular}

${ }^{a} \mathrm{~N}-\mathrm{H}$ distances were kept frozen at their standard neutron values ${ }^{17 \mathrm{~b}}$.

${ }^{\mathrm{b}}$ Experimentally-derived charge density and its Laplacian as computed at the bond critical point (bcp) along the atomic interaction line ${ }^{29}$ linking $\mathrm{H}$ and $\mathrm{O}$ atoms. Estimated standard deviations provided by the $\mathrm{XD} 2006^{28}$ program are also shown.

${ }^{\mathrm{c}}$ Symmetry operation that generates the acceptor $\mathrm{O}$ atom.

The two N1-H...O 1 bonds forming the cyclic HB pattern (Figures 1,2) are very similar in terms of geometrical and topological descriptors, though they are not symmetry-related. One could explain such similarity in terms of a pseudo-inversion operator, e.g. located at the geometric centre of the hydrogen-bonded 8-membered ring, $\quad[-\mathrm{N} 1 \mathrm{~A}-\mathrm{HN} 1 \mathrm{~A} \cdots \mathrm{O} 1 \mathrm{~B}-\mathrm{C} 1 \mathrm{~B}-\mathrm{N} 1 \mathrm{~B}-\mathrm{HN} 1 \mathrm{~B} \cdots \mathrm{O} 1 \mathrm{~A}-\mathrm{C} 1 \mathrm{~A}-]$. However, deviations from $C_{\mathrm{i}}$ symmetry are significant within the $\mathrm{AB}_{\mathrm{i}}$ adduct (see Section $\mathrm{S} 5, \mathrm{SI}$ ). The symmetry breaking in the zone of the pyrazolidinone rings is due to the mutual orientation of the two molecules, as facing amide groups are not coplanar. The planes passing through atoms H1$\mathrm{N} 1-\mathrm{C} 1-\mathrm{O} 1$ in A and B molecules are tilted by $36.31(3)^{\circ}$ with respect to each other. This is not due to conformational differences, as their 5-membered rings are almost identical (see Sections 3.2.1, and S5, SI). Rather, the ASU adopts an asymmetric configuration as a whole: no obvious pseudoinversion operators can be recognized, not even correlating pyrazolidinone groups. This implies that the similarity showed by the $\mathrm{N} 1-\mathrm{H} 1 \cdots \mathrm{O} 4 \mathrm{HBs}$ has a purely chemical origin, i.e. it is due to the 
similarity, in terms of bond geometries and hybridization states, of the interacting amide groups. Significant differences become instead evident when the N3-H‥O4 bonds are compared (Table 2), as the $\mathrm{N} 3 \mathrm{~A}-\mathrm{HN} 3 \mathrm{~A} \cdots \mathrm{O} 4 \mathrm{~B} \mathrm{HB}$ is clearly weaker than its $\mathrm{N} 3 \mathrm{~B}-\mathrm{HN} 3 \mathrm{~B} \cdots \mathrm{O} 4 \mathrm{~A}$ analogue. This is due to a less favourable geometry of the first HB, because of the conformationally-driven differences in the mutual orientation of the N3 donor and $\mathrm{O} 4$ acceptor atoms in the A and B enantiomers (see Section 3.2.1 below).

These packing motifs are consistent with a truly polar crystal structure. ${ }^{6}$ The resulting dipole moments from all the A and B molecules in the unit cell are oppositely directed and run along the polar $c$ axis. From the Mulliken partitioning of the quantum mechanical charge density in the bulk (Section 2.2) at the $100 \mathrm{~K}$ experimental geometry, it emerges that the four B molecules in the unit cell provide a dipole contribution as large as $10.7 \mathrm{D}$ along [ $\left.\begin{array}{lll}0 & 0 & 1\end{array}\right]$, which is counteracted by that

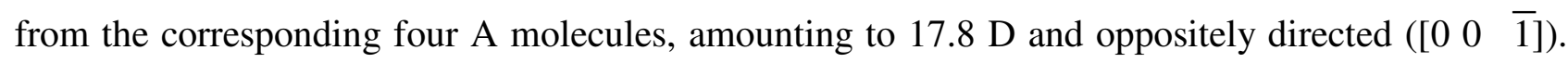
Overall, the total in-cell dipole module amounts to 7.1 D.

3.1.2 Hirshfeld surface fingerprint plots. Figure 3 shows the Hirshfeld surface fingerprint plots $^{39}$ of the independent conformers in PYRAC at $T=100(2) \mathrm{K}$. By definition, a Hirshfeld surface surrounding a given molecule encloses the region of space inside which the promolecular density ${ }^{40}$ exceeds that due to any other molecule. A fingerprint plot is a map of the fraction of points on the Hirshfeld surface that exhibit specific $d_{\mathrm{i}}$ and $d_{\mathrm{e}}$ values, the latter being the closest distances of nuclei inside $\left(d_{\mathrm{i}}\right)$ and outside $\left(d_{\mathrm{e}}\right)$ the volume enclosed by the surface itself. Fingerprint plots thus encode information about the intermolecular interactions throughout the nearest environment of each molecule in the ASU. They are suitable to study polymorphism ${ }^{41}$ and/or structures with $Z>1$, i.e. where more than one formula unit is present in the ASU. ${ }^{14,39 b}$
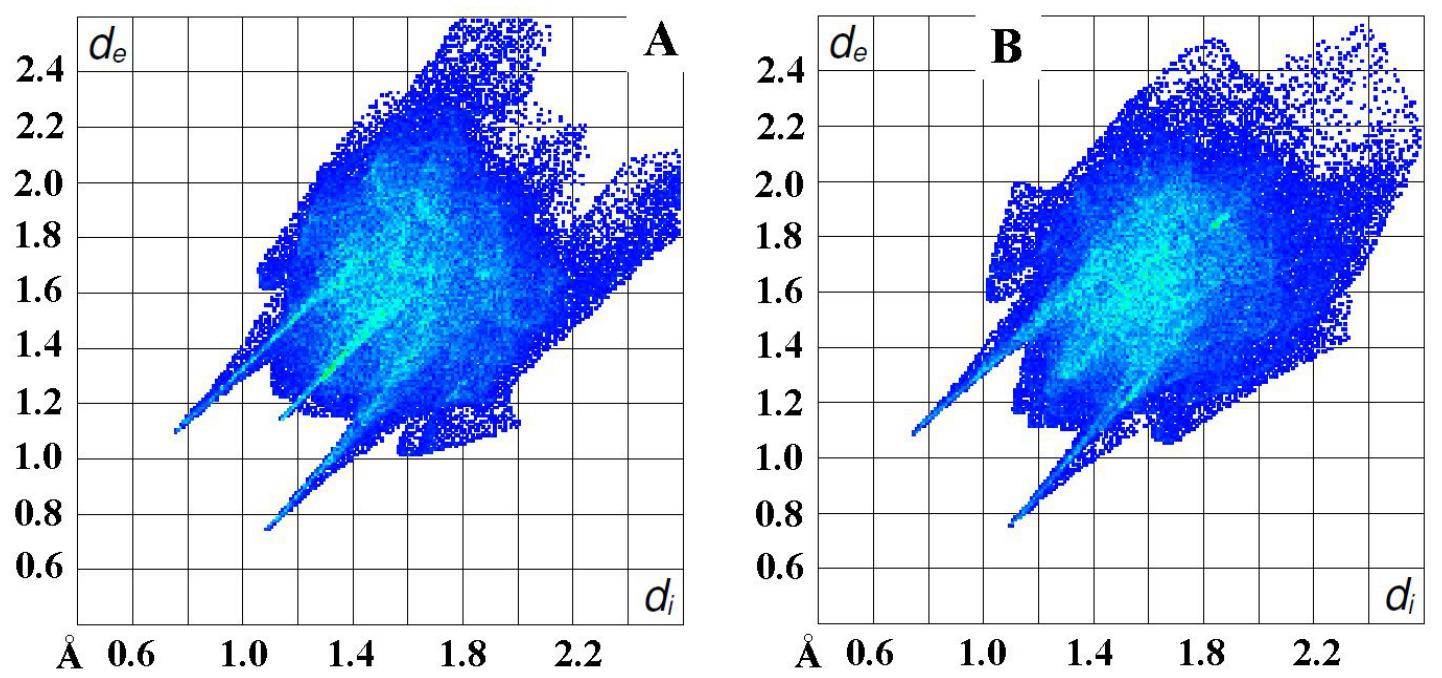

Figure 3. Colour online. Hirshfeld surface fingerprint plots of the nearest internal distance $\left(d_{\mathrm{i}}\right)$ versus the nearest external distance $\left(d_{\mathrm{e}}\right)$ for the two independent conformers A (left) and B (right) of PYRAC at $T=100 \mathrm{~K}$. The colours represent the number of points which share the same $d_{\mathrm{i}}, d_{\mathrm{e}}$ 
coordinate (light blue: many; dark blue: few). The graphics were produced using Crystal Explorer v2.1..$^{42}$

From Figure 3, the following analogies and differences between the two conformers are noted. (i) Two long and sharp lateral spikes are evident in both the diagrams, with minimum $d_{\mathrm{i}}+d_{\mathrm{e}} \approx 1.8-1.9$ $\AA$. This is the signature of the four independent short NH $\cdots \mathrm{O}$ hydrogen bonds above discussed. The fact that the spikes are almost identical and symmetric with respect to the main diagonal of either diagram, despite the asymmetric nature of the ASU (see above), remarks that all the strong HBs are set up between the same pairs of donor and acceptor molecules. (ii) A narrow light blue spike along the main diagonal with minimum $d_{\mathrm{i}}+d_{\mathrm{e}} \approx 2.2 \AA$ is appreciable in the plot of the conformer $\mathrm{A}$, but not in that of the conformer B. This feature is due to head-to-head $\mathrm{H} \cdots \mathrm{H}$ contacts, such as nearly linear $\mathrm{C}-\mathrm{H} \cdots \mathrm{H}-\mathrm{C}$ arrangements, ${ }^{39}$ between neighbouring molecules. It reveals that the A molecule experiences a slightly more crowded environment than B. On the contrary, (iii) the latter shows a more localized narrow light blue spot at $d_{\mathrm{i}}=d_{\mathrm{e}} \approx 1.8 \AA$. This is attributable to a single $\mathrm{C} \cdots \mathrm{C}$ contact between $\mathrm{C} 7 \mathrm{~B}[\mathrm{x}, \mathrm{y}, \mathrm{z}]$ and $\mathrm{C} 1 \mathrm{~B}[0.5+\mathrm{x},-\mathrm{y}, \mathrm{z}]$ carbonyl atoms, that belong to a couple of $\mathrm{B}$ conformers enantiomerically related through an $a$ glide plane (Figure S13 in the Supplementary Materials). Being located at the characteristic $d_{\mathrm{i}}$ and $d_{\mathrm{e}}$ coordinates of the $\pi \cdots \pi$ stacking interactions in aromatic hydrocarbons, this feature implies some kind of such interactions between the pyrazolidinone and ester groups of stacked B molecules. (iv) A couple of asymmetric lateral wings at minimum $d_{\mathrm{i}}+d_{\mathrm{e}} \approx 2.5-2.7 \AA$ are evident in both the plots. These wings indicate $\mathrm{C}-\mathrm{H} \cdots \pi$ interactions that typically appear when an H-bearing terminal group is oriented so that it is roughly equidistant from a planar hydrocarbon ring. ${ }^{39}$ As for the present case, the A phenyl can accept a C$\mathrm{H} \cdots \pi$ contact from the $\mathrm{H} 3 \mathrm{~B}$ tertiary hydrogen, that is roughly equidistant from the $\mathrm{C} 11 \mathrm{~A}-\mathrm{C} 16 \mathrm{~A}$ carbon atoms $\left(\left\langle\mathrm{d}_{\mathrm{H} 3 \mathrm{~B} \cdots \mathrm{C}}\right\rangle=2.88(9) \AA\right)$. The phenyl group of the $\mathrm{B}$ conformer, on the other hand, is a less efficient acceptor, as the analogue $\mathrm{C} 3 \mathrm{~A}-\mathrm{H} 3 \mathrm{~A} \cdots \pi$ interaction has a more unfavourable geometry, with uneven and, on average, longer $\mathrm{H} \cdots \mathrm{C}$ distances $\left(<\mathrm{d}_{\mathrm{H} 3 \mathrm{~A} \cdots \mathrm{C}}>=3.2(2) \AA ̊ \AA\right.$; Figure S14, SI). 362833

\subsection{Molecular recognition.}

3.2.1. Conformers present in the solid state. The PYRAC hydrocarbon backbone is dominated by $\sigma$-bonds. Therefore, this compound has a significant conformational flexibility that leads to important conformational differences between the A and B molecules, contributing to avert the ASU to be centrosymmetric (see Section 3.1.1 above). Figure 4 shows the main differences between two homochiral C3(S), C6(R) conformers once superimposing the two carbammate moieties, sited near the respective centres of mass. 


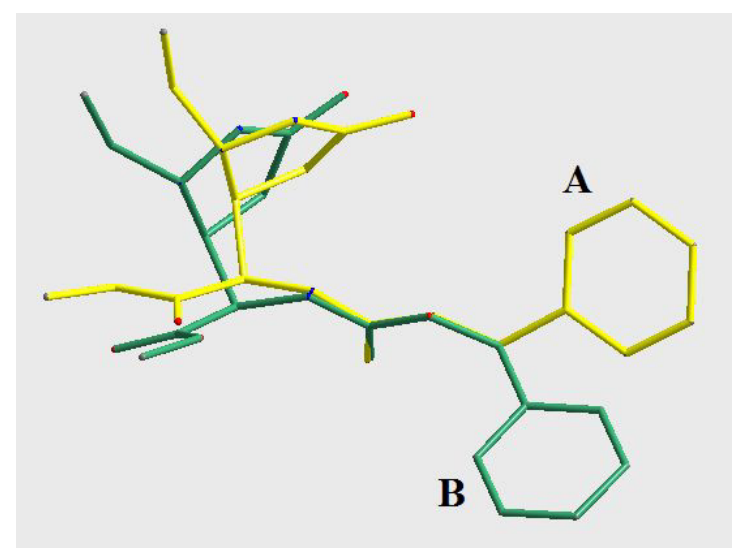

Figure 4. Colour online. Superposition of two A (yellow) and B (green) homochiral conformers at their solid-state geometries, as determined by the diffraction experiment at $T=100 \mathrm{~K}$. Hydrogen atoms have been omitted for the sake of clarity.

Interestingly, different functional groups are rigidly displaced on going from one conformer to the other. In particular, the 5-membered pyrazolidinone rings adopt the same envelope-like configuration at the $\mathrm{C} 3$ atom, with puckering coordinates ${ }^{43} Q(2)_{\mathrm{A}}=0.262, \phi(2)_{\mathrm{A}}=257.11 \mathrm{deg}$ for the A backbone and $Q(2)_{\mathrm{B}}=0.208, \phi(2)_{\mathrm{B}}=77.51 \mathrm{deg}$ for the $\mathrm{B}$ one. Note that $\phi(2)_{\mathrm{A}}$ and $\phi(2)_{\mathrm{B}}$ are identical within $0.5 \mathrm{deg}$, apart a $\pm \pi$ phase shift accounting for the change in the absolute configuration. In summary, the most relevant differences concern the mutual orientations of the phenyl rings ${ }^{44}$ and ester chains. In particular, the latter are rotated by almost $180^{\circ}$ around the C6-C7 bond, with torsion angles $\tau_{\mathrm{O} 2-\mathrm{C} 7-\mathrm{C} 6-\mathrm{N} 3}$ being as large as -0.28(9) deg in $\mathrm{A}$ and 176.03(7) deg in $\mathrm{B}$ homochiral molecules. The two in-crystal conformations lie very close in energy, with the A being more stable than the B conformation by just $1.5 \mathrm{kcal} \cdot \mathrm{mol}^{-1}$ at the B3LYP 6-311G(p,d) theory level.

3.2.2. DFT conformational dynamics. To gain insights into the formation of the $\mathrm{AB}_{\mathrm{i}}$ building block, it is instructive to explore the pseudorotatory path for the $\mathrm{A} \leftrightarrow \mathrm{B}$ interconversion in vacuo (Section 2.4 above and S6, SI). We found that the A $\leftrightarrow$ B interconversion takes place through a couple of competing reaction paths. Both of them occur in two steps and involve two new stable minima, not present in the crystal (hereinafter, 'G' and 'M'). Figure 5 shows the energy profile for the $\mathrm{A} \leftrightarrow \mathrm{B}$ reaction through the various stationary points it crosses. The key substituents involved in the main conformational changes are just the benzyl and the methylester (see also Sections S5 and S6, SI). Both these groups can rotate around a specific $\sigma$-bond, i.e. O5-C10 (benzyl) and C6-C7 (ester). The rotation of the aromatic system is almost free, with the corresponding $\approx 90^{\circ}$ wide sweeping being associated to very small activation barriers (TS1 and TS4). The rotation of the methylester group is instead more difficult, as it proceeds through the high energy transition states TS2 and TS3. If the benzyl group rearranges first, the $\mathrm{A} \leftrightarrow \mathrm{M} \leftrightarrow \mathrm{B}$ path is followed: the first elementary act 
implies evolution into the transition state TS1, that is similar to the starting point $\mathrm{A}$ apart $\mathrm{a} \approx 90^{\circ}$ rotation of the benzyl itself (Figure S11a, SI).

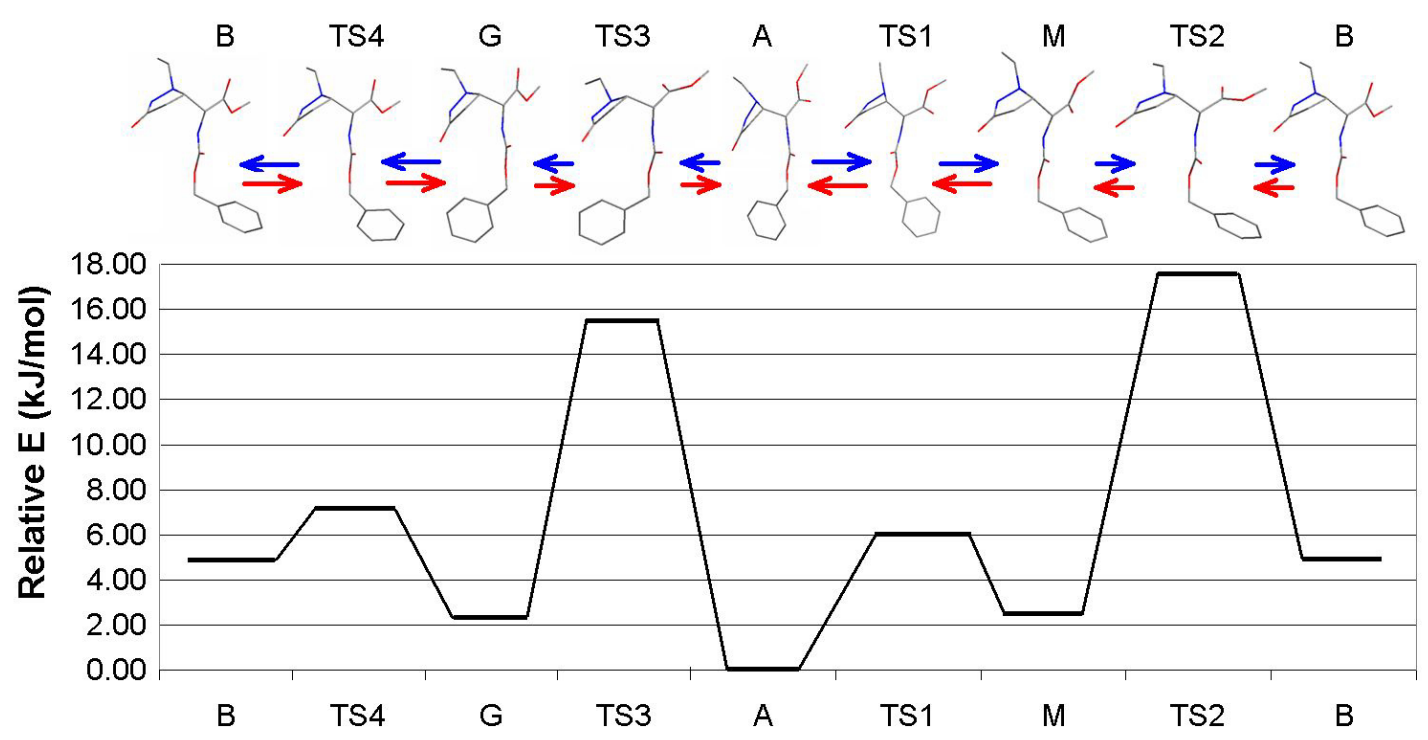

Figure 5. Colour online. In vacuo energy profile $\left(\mathrm{kJ} \cdot \mathrm{mol}^{-1}\right)$ at the B3LYP $6-311 \mathrm{G}(\mathrm{d}, \mathrm{p})$ level of theory $v s$. the reaction coordinate, taking the energy of the most stable A conformer as reference. All the geometries were fully optimized and corrections for zero-point energy were taken into account. Top: backbone structures (no hydrogen atoms shown) of stable conformers and transition state (TS) geometries. Shorter arrows correspond to slower elementary acts.

Then, PYRAC falls into the high-energy M minimum: now the benzyl is oriented as in the final B state, while the methylester chain is kept essentially unchanged with respect to the starting stationary point (see also Figure S10d, SI). The second act starts with a more difficult $90^{\circ}$ rotation of the methylester chain around the C6-C7 bond, until the late transition state TS2 is reached (Figure S11b, SI). Eventually, the rotation of the ester group is completed and TS2 evolves into the B conformer.

As for the second available reaction path, it proceeds through the $\mathrm{G}$ intermediate following the $\mathrm{A} \leftrightarrow \mathrm{G} \leftrightarrow \mathrm{B}$ mechanism. The latter is equivalent to the $\mathrm{A} \leftrightarrow \mathrm{M} \leftrightarrow \mathrm{B}$ one, but the order of the conformational changes above described is now reversed.

Table 3. Kinetic constants at $T=298 \mathrm{~K}$ for the elementary acts involved in the $\mathrm{A} \leftrightarrow \mathrm{B}$ interconversion mechanism, as estimated from quantum mechanical simulations in vacuo at the B3LYP 6-311G(p,d) level of theory. For each step, the height of the potential barrier is also shown.

\begin{tabular}{c|r|rr} 
Elementary act & Label & $k\left(\times 10^{10}\right) / \mathrm{s}^{-1}$ & $\Delta E / \mathrm{kJ} \cdot \mathrm{mol}^{-1}$ \\
\hline $\mathrm{A} \leftrightarrow \mathrm{M} \leftrightarrow \mathrm{B}$ path & & & \\
$\mathrm{A} \rightarrow \mathrm{M}$ & $k_{1}$ & 55.13 & 6.00 \\
$\mathrm{M} \rightarrow \mathrm{A}$ & $k_{-1}$ & 149.80 & 3.52 \\
$\mathrm{M} \rightarrow \mathrm{B}$ & $k_{2}$ & 1.39 & 15.11 \\
$\mathrm{~B} \rightarrow \mathrm{M}$ & $k_{-2}$ & 3.65 & 12.73 \\
$\mathrm{~A} \leftrightarrow \mathrm{G} \leftrightarrow \mathrm{B}$ path & & & \\
$\mathrm{A} \rightarrow \mathrm{G}$ & $k_{3}$ & 1.21 & 15.46 \\
$\mathrm{G} \rightarrow \mathrm{A}$ & $k_{-3}$ & 3.18 & 13.07 \\
$\mathrm{G} \rightarrow \mathrm{B}$ & $k_{4}$ & 90.40 & 4.77 \\
$\mathrm{~B} \rightarrow \mathrm{G}$ & $k_{-4}$ & 245.10 & 2.30 \\
\hline
\end{tabular}


This implies that the slow step is associated to the early transition state, not to the late one. Accordingly, the intermediate stationary points $\mathrm{G}$ and $\mathrm{M}$ differ from each other from the mutual orientation of both the ester and the benzyl groups (Figure S12, SI). Table 3 shows the kinetic constants at $T=298 \mathrm{~K}$ for the various elementary acts described above and computed from the Eyring equation. As expected on the basis of the energy profile in Figure 5, the constants associated to the rotation of the methylester, $k_{ \pm 1}, k_{ \pm 4}$, are from one to two orders of magnitude greater than those of the motion of the benzyl, $k_{ \pm 2}, k_{ \pm 3}$. In any case, the overall process tends to be directed towards the most stable product, A, as the reverse reactions always have at least twice as large kinetic constants than the corresponding direct reactions.

3.2.3 Competing synthons. We focus here just on fully centrosymmetric possible synthons ${ }^{45}$ to gain insights on the neat preference for the polar space group exhibited by PYRAC. Starting from the four stable A, M, G and B geometries found in vacuo, we built up centrosymmetric pairs, trying to set up as many as possible favourable $\mathrm{NH} \cdots \mathrm{O}$ and $\mathrm{CH} \cdots \mathrm{O} \mathrm{HB}$ contacts while avoiding evident steric clashes. We employed the Clpdim routine in the CLP program package ${ }^{33}$ to compute the interaction energies of the corresponding pairs. CLP-derived energies were then minimized by manually applying mutual translations and rotations to the interacting molecules, within the constraint of preserving the inversion symmetry of the pair. Eventually, the most stable adducts were fully optimized in vacuo at the B3LYP 6-311G(p,d) level of theory. A vibrational analysis was also performed to ensure that the relaxed geometries of each pair truly corresponded to stationary points on the potential energy surface (PES).

Figure 6 displays the structures found through this procedure. Cyclic HB patterns are always set up, but only when they involve facing amide groups as in the solid state (see Section 3.1 above), the resulting pair has interaction energy $E_{\mathrm{int}}<<$. Pairs lacking this specific interaction are always weakly bonded, with interaction energies being equal to zero within $1-2 \mathrm{kcal} \cdot \mathrm{mol}^{-1}$. This recognition pattern is possible in most of the $\mathrm{XX}_{\mathrm{i}}$ adducts. $\mathrm{GG}_{\mathrm{i}}$ is the only exception, as a steric clash among phenyl rings and ethyl chains would result if the 5-membered rings of two $G$ conformers are brought too close to each other. Low $\left|E_{\text {int }}\right|$ estimates (Figure 6) imply that thermal excitations and collisions with solvent are expected to carry enough energy to put apart the corresponding pairs, at ordinary temperatures. Therefore, formation of symmetric or asymmetyric HBs between heterochiral pyrazolidinone rings in PYRAC appears to be a prerequisite for fully stable supramolecular synthons. Overall, 4 building blocks $\left(\mathrm{AA}_{\mathrm{i}}(2), \mathrm{BB}_{\mathrm{i}}, \mathrm{AB}_{\mathrm{i}}, \mathrm{MM}_{\mathrm{i}}(2)\right)$ should be available for PYRAC crystallization. However, their formation is not equally probable, as the accessible molecular stationary points are not evenly populated. 
Supposing that at thermal equilibrium the available conformers are Boltzmann-distributed, the population probabilities, $p_{\mathrm{X}}$, of the $\mathrm{A}, \mathrm{M}, \mathrm{G}$ and $\mathrm{B}$ minima as obtained from the partition function analysis are $0.5293(\mathrm{~A}), 0.1948(\mathrm{M}), 0.2016(\mathrm{G})$ and $0.0743(\mathrm{~B})$ at $T=298 \mathrm{~K}$.

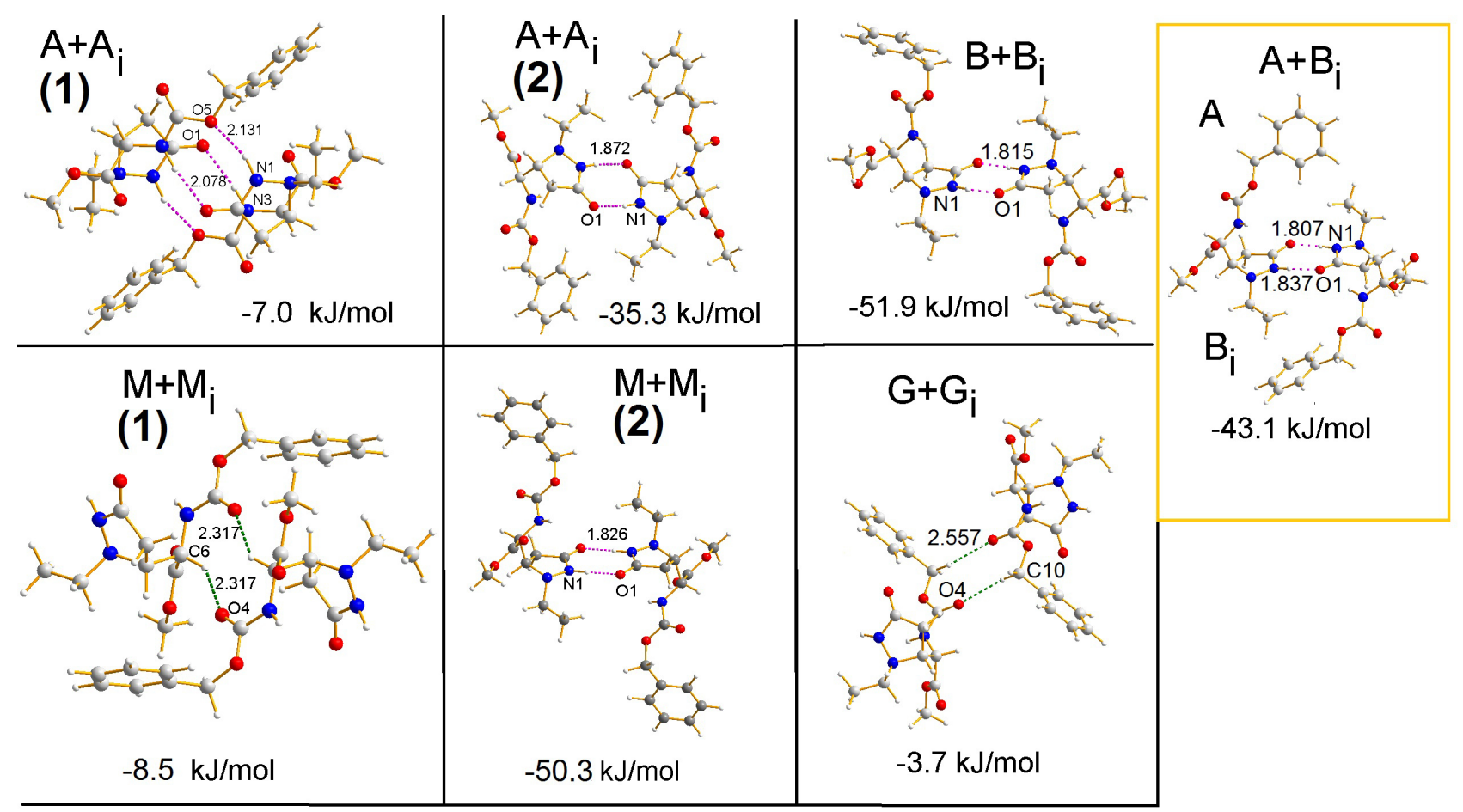

Figure 6. Stationary points on the PES corresponding to centrosymmetric pairs of various PYRAC conformers, as found by in vacuo DFT simulations. For each structure, BSSE- and zero-point corrected quantum mechanical interaction energies are given. HB contacts are highlighted as purple $(\mathrm{NH} \cdots \mathrm{O})$ and green $(\mathrm{CH} \cdots \mathrm{O})$ dotted lines. Related bond lengths (in $\AA$ ) and labels of donor and acceptor atoms involved in the interaction are also specified. A yellow box encloses the relaxed structure of the $\mathrm{AB}_{\mathrm{i}}$ synthon.

These values correspond to the fraction of time spent by each molecule in the liquor in the conformation $\mathrm{X}(\mathrm{X}=\mathrm{A}, \mathrm{M}, \mathrm{G}$ or $\mathrm{B})$ at equilibrium. At the end of the chemical synthesis, an equimolar quantity of PYRAC enantiomers is obtained. Therefore, the probability that two specific heterochiral conformers $\mathrm{X}$ and $\mathrm{Y}_{\mathrm{i}}$ will interact is given by the product of the corresponding population probabilities ( $\mathrm{Y}$ may be equal to $\mathrm{X}$ or not, Figure $7 \mathrm{a}$ ). Degeneracy must also be taken into account, as a $\mathrm{XY}_{\mathrm{i}}$ adduct is energetically equivalent to its enantiomer $\mathrm{X}_{\mathrm{i}} \mathrm{Y}$.

The most populated A conformer dominates the meeting process, as A-containing pairs occur more frequently. However, the interaction probabilities shown in Figure 7a are computed from a purely stochastic perspective, i.e. they measure the likelihood two different heterochiral conformers meet each other in the liquor. Further scaling factors should be taken into account to model the 'effectiveness' of each collision to produce both a kinetically and thermodynamically stable adduct. Reorientation of the conformers taken as rigid bodies, together with internal 
relaxation of individual molecules, might have a not-negligible influence on the probability that a specific self-recognition process be effectively observed.
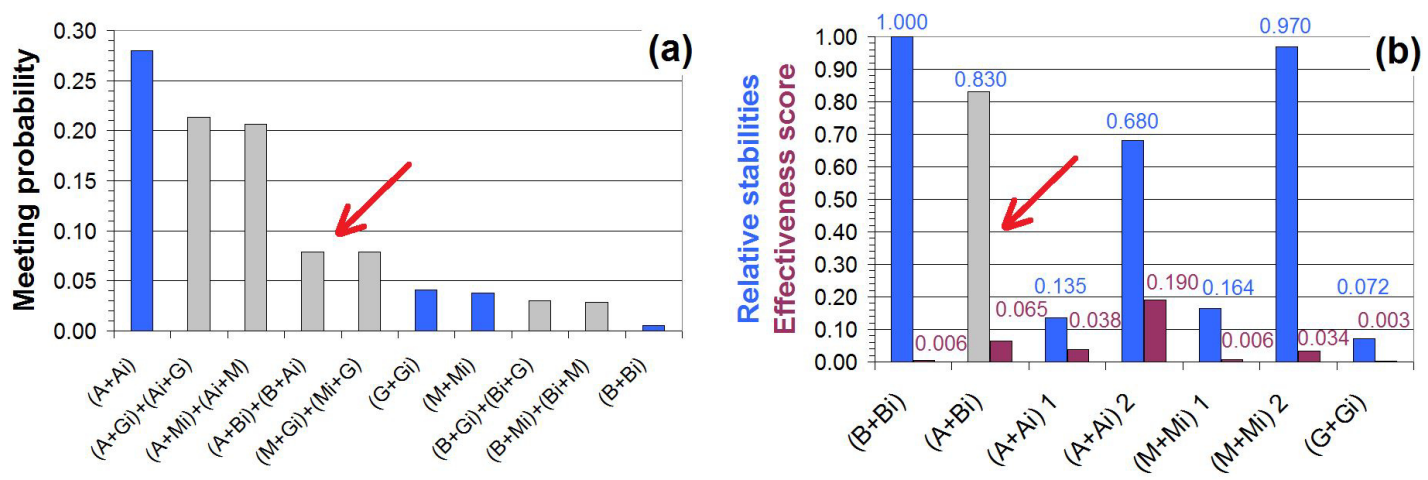

Figure 7. Colour online. (a) Meeting probabilities of different conformers (A, M, G, B: see text) with their enantiomers. Blue: $C_{\mathrm{i}}$ pairs. Grey: $C_{1}$ pairs. The red arrow highlights the $\mathrm{AB}_{\mathrm{i}}$ pair. (b) Blue $\left(C_{\mathrm{i}}\right)$ and grey $\left(C_{1}\right)$ bars: Relative $E_{\mathrm{int}}$ of selected adducts as computed from in vacuo DFT simulations, with respect to the most stable $\mathrm{BB}_{i}$ pair. Purple bars: Overall effectiveness score for selected pairs (see text), obtained by scaling the meeting probabilities for the relative $E_{\text {int }}$ values. See Figure 6 for the meaning of the labels on the $x$ axis.

In this context, it is reasonable to rescale the crude meeting probabilities ${ }^{46}$ shown in Figure 7a by means of the relative stabilities of the corresponding molecular pairs, for example by taking the most stable $\mathrm{BB}_{\mathrm{i}}$ one as a reference (Figures 6 and $7 \mathrm{~b}$ ). ${ }^{47}$ In this way, the various adducts can be ranked (purple bars in Figure 7b) as a function of their propensities to act as effective synthons. The higher the final score, the greater will be the likelihood that the corresponding synthon will form frequently enough and be stable enough to start growing a crystal embryo before being destroyed by thermal fluctuations and/or collisions with its surroundings. Within this simple model, the AA $(2)$ and $\mathrm{AB}_{\mathrm{i}}$ pairs have the highest scores (0.190 and 0.065 , respectively), while other possible centrosymmentric adducts are significantly less favourite $\left(\mathrm{AA}_{\mathrm{i}} 1: 0.0038 ; \mathrm{MM}_{\mathrm{i}} 2: 0.0034 ; \mathrm{BB}_{\mathrm{i}}\right.$ and $\mathrm{MM}_{\mathrm{i}}$ 1: 0.006,, GGi: 0.003).

In summary, the likelihood of specific intermolecular recognition processes in the very first stages of the nucleation process is influenced by various factors, such as the relative stability of the accessible conformers and the populations of the corresponding stationary points on the PES, including those of the high-energy ones that are crossed during the $\mathrm{A} \leftrightarrow \mathrm{B}$ interconversion path. As a consequence, looking just at the relative stabilities of possible competing synthons to determine which pair will eventually prevail in the solid state is not sufficient. On the other hand, knowledge of the conformational space allows for predicting, at least in principle, how the whole recognition process will be influenced upon changing some of the boundary conditions of the liquor. For example, in the present case, it is reasonable that a decrease of $T$ should hamper the formation of the $\mathrm{AB}_{\mathrm{i}}$ synthon by further depopulating the high-energy $\mathrm{B}$ minimum, so favouring the formation of $\mathrm{AA}_{\mathrm{i}}$ pairs. 


\subsection{Centrosymmetric PYRAC polymorphs?}

3.3.1 Looking for competing centrosymmetric polymorphs. structure will be eventually observed depends on the often quite subtle interplay among various classes of non-covalent interactions that are set up in the crystal, such as the HBs, the van der Waals-like or the electrostatics interactions and the onset of Pauli repulsions. ${ }^{48}$ To understand why a non-centrosymmetric synthon, and a fortiori a non-centrosymmetric structure, is ultimately preferred in PYRAC over alternative centrosymmetric synthons, one has to explore hypothetical centrosymmetric polymorphs that employ $C_{\mathrm{i}} \mathrm{XX}_{\mathrm{i}}(\mathrm{X}=\mathrm{A}, \mathrm{M}, \mathrm{G}, \mathrm{B})$ rather than the $C_{1} \mathrm{AB}_{\mathrm{i}}$ building blocks. To this end, translational symmetry was applied to the in vacuo optimized adducts discussed in the previous Section (Figure 6) by means of the Clpoly program in the CLP package ${ }^{33}$ (see Section 2.3 above). Figure 8 shows the best results so obtained, in terms of lattice cohesive energies and crystal density. The corresponding numerical entries, together with the refined unit cells and individual energy contributions, can be found in Table S6, SI.

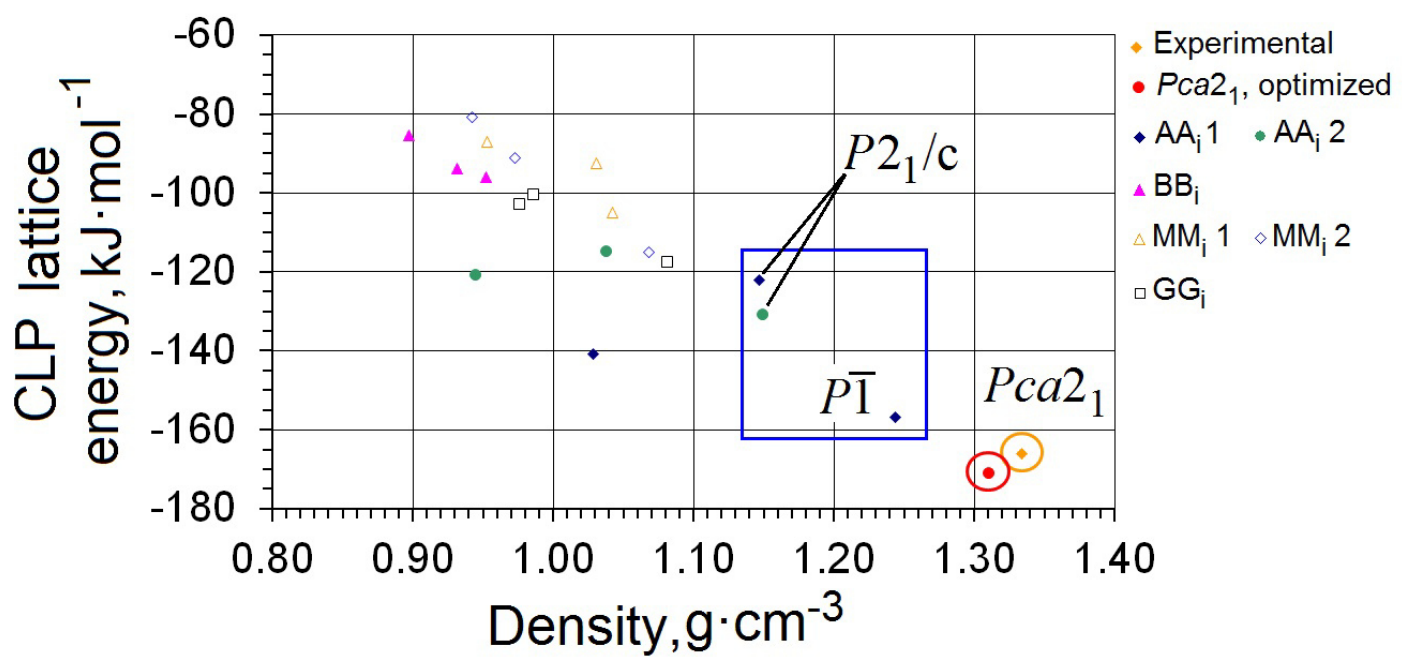

Figure 8. Colour online. CLP lattice energies, $E_{\text {latt }}, v s$. the crystal density, as evaluated for various PYRAC polymorphs. A yellow circle highlights the polar non-centrosymmetric Pca2 $2_{1}$ structure at the $100 \mathrm{~K}$ X-ray geometry, while a red circle encompasses the same structure after optimization. The blue box encloses structures with packing coefficient $0.6<C_{\text {pack }}<0.7$, comparable to that found experimentally $\left(C_{\text {pack }}=0.7\right)$.

Several stable structures were found in space groups $P 2_{1} / c, P \overline{1}$ and $P b c a$, while the $C 2 / c$ group provided inefficient packing hypotheses, with an average packing coefficient ${ }^{10 \mathrm{~b}}<C_{\text {pack }}>=$ 0.41(4). In general, the more favourable is the lattice energy, the higher the density of the corresponding crystal. However, just three structures (enclosed in a blue rectangle in Figure 8) have $C_{\text {pack }}>0.6$ that can be considered as the lower limit for the packing efficiency observed in most organic crystals. ${ }^{10 \mathrm{~b}}$ These forms derive exclusively from synthons of the $\mathrm{AA}_{\mathrm{i}}$ type (Figure 6), not from the most stable $\mathrm{BB}_{\mathrm{i}}$ adduct (full purple triangles in Figure 8). They are also comparable in 
terms of lattice energies, densities and $C_{\text {pack's }}$ with respect to the experimental $P c a 2_{1}$ crystal (yellow circle in the Figure), even though the latter shows a higher lattice stability. This is even more evident if the $P c a 2_{1}$ structure is made fully comparable with the simulated centrosymmetric ones upon relaxing its lattice parameters and the ASU orientation through the CLP force field (red circle in Figure 8).

Full-electron periodic quantum mechanical optimizations at the B3LYP 6-31G(p,d) theory level were performed for both the experimental structure and those forms with $C_{\text {pack }}>0.6$. Then, the total crystal cohesive energies, $E_{\text {coh}}$, of the fully relaxed structures can be computed through ${ }^{14}$ :

$$
E_{\text {coh }}=E_{\text {bulk }}-\sum_{i} E_{\text {iso }}(i)-\sum_{i} E_{\text {rel }}(i)+\sum_{i} E_{\mathrm{BSSE}}(i)
$$

where $E_{\text {bulk }}$ is the total electron energy of the unit cell, $E_{\mathrm{iso}}(i)$ is the energy of each $i$ th isolated molecule at its solid-state conformation, $E_{\text {rel }}(i)$ is the relaxation energy (it is a negative term accounting for the difference between the energies of a single molecule, relaxed in vacuo, and of the same molecule at the solid-state relaxed geometry) and $E_{\mathrm{BSSE}}(i)$ is the correction for basis-set superposition error. Summations run over the content of the unit cell.

From Table 4, it is clear that the most stable structure at $T=0 \mathrm{~K}$ is invariably the polar $P c a 2_{1}$ one. The latter is predicted to be less dense than the phase experimentally found at finite temperature. This is due to $\mathrm{a} \approx 5 \%$ increase in length of the three cell axes and is the consequence of neglecting dispersive (attractive) interactions by ground-state DFT methods. ${ }^{49}$ Similar results were also found when organic crystals were simulated within the DFT GGA approximation. ${ }^{50} 3314$ In any case, the present calculations are just approximations of the true thermodynamic crystal stability $^{51}$ and clearly do not claim to accurately reproduce the experimental structure. Such a task would require a finite-temperature modeling of the electronic and phononic structures of both the hypothetic and real crystal forms, in tandem with post-DFT quantum methods. Nonetheless, our calculations represent a convenient tool for ranking the energies of the polymorphs on a relative scale. In this respect, the $\mathrm{P} \overline{1}$ form turns out to be the least stable one once that all the corrective terms are applied. This is due to the unfavourable $E_{\text {rel }}$ and $E_{\mathrm{BSSE}}$ terms, which are both significantly greater in this than in the $P 2{ }_{1} / c$ structures. The BSSE correction depends on the final relaxed geometry of the whole structure and more closely packed arrangements usually imply higher BSSE correction terms. The relaxation energy, on the other hand, allows for estimating the importance of the crystal field in forcing the molecular backbone to assume a high-energy geometry. While A and B molecules within the $P c a 2_{1}$ structure are already close to their gas-phase minima (Figure S16, SI), the A conformers of the other polymorphs are subject to larger rearrangements in the pyrazolidinone region when they are optimized in vacuo at B3LYP 6-31G(p,d) level of theory. 
Table 4. Total crystal cohesive energies, $E_{\text {coh }}$, as computed for optimized $(T=0 \mathrm{~K})$ solid-state structures of PYRAC. All the calculations were performed at the B3LYP 6-31G(p,d) level of theory. The entries are given in $\AA$, deg, $\mathrm{g} \cdot \mathrm{cm}^{-3}$ and $\mathrm{kJ} \cdot \mathrm{mol}^{-1}$.

\begin{tabular}{|c|c|c|c|c|c|c|c|c|c|}
\hline Lattice & Synthon $^{a}$ & $\begin{array}{c}\text { Cell } \\
\text { edges }\end{array}$ & $\begin{array}{c}\text { Cell } \\
\text { angles }\end{array}$ & Density & $C_{\text {pack }}{ }^{\mathrm{b}}$ & $\begin{array}{l}E_{\text {bulk- }} \\
\sum_{\mathrm{i}} E_{\text {iso }}\end{array}$ & $E_{\mathrm{BSSE}^{\mathrm{c}}}$ & $E_{\text {rel }}$ & $E_{\mathrm{coh}}$ \\
\hline $\mathrm{Pca}_{1}$ & $\mathrm{AB}_{\mathrm{i}}$ & $\begin{array}{c}15.1553 \\
9.6209 \\
965084\end{array}$ & $\begin{array}{l}90.00 \\
90.00\end{array}$ & 1.148 & 0.7 & -210.61 & 124.00 & -41.93 & -44.68 \\
\hline$P \overline{1}$ & $\mathrm{AA}_{\mathrm{i}} 1$ & $\begin{array}{c}26.5984 \\
9.6204 \\
16.9671 \\
17.5530\end{array}$ & $\begin{array}{c}90.00 \\
66.32 \\
109.54 \\
49.07\end{array}$ & 1.114 & 0.6 & -137.37 & 106.63 & -77.19 & 46.45 \\
\hline$P 2_{1} / c$ & $\mathrm{AA}_{\mathrm{i}} 1$ & $\begin{array}{c}15.1907 \\
13.2840 \\
11.7221 \\
14.003\end{array}$ & $\begin{array}{c}90.00 \\
105.21 \\
90.00 \\
90.00\end{array}$ & 0.975 & 0.5 & -71.62 & 44.94 & -17.32 & -9.36 \\
\hline$P 2_{1} / c$ & $\mathrm{AA}_{\mathrm{i}} 2$ & $\begin{array}{c}14.003 \\
13.6682 \\
11.4972\end{array}$ & $\begin{array}{l}90.00 \\
90.35 \\
90.00\end{array}$ & 1.012 & 0.6 & -81.05 & 43.64 & -16.32 & -21.09 \\
\hline
\end{tabular}

${ }^{\text {a }}$ See Figure 6 for the meanings of the various labels.

${ }^{\mathrm{b}}$ Packing coefficient (adimensional), defined as the ratio between the total volume occupied by molecules ${ }^{10 \mathrm{~b}}$ and the cell volume.

${ }^{c}$ A $7 \AA$ A cutoff was employed to generate ghost atoms ${ }^{30 a}$. For each structure, this limit was set by looking for the distance above which the BSSE correction was constant.

As expected (see Sections S5 and S6, SI), the benzyl group is relatively free to rotate: for example, in the $P \overline{1}$ simulated polymorph a $\approx 17^{\circ}$ wide rotation occurs around the $\mathrm{C} 10-\mathrm{O} 5$ bond with respect to the in vacuo arrangement.

It is also worth noting that the Pca2 $2_{1}$ optimized structure resembles the experimental one. Again, the major changes in the A and B conformers in the two crystalline phases involve small adjustments, not exceeding $\approx 10-15^{\circ}$, of the torsion angle $\tau(\mathrm{C} 11-\mathrm{C} 10-\mathrm{O} 5-\mathrm{C} 9)$ describing the orientation of the benzyl system with respect to the carbammate moiety. These differences do not qualitatively influence the main HB patterns found by the X-ray analysis (see Figure 2 and Tables $\mathrm{S} 3$ and $\mathrm{S} 4, \mathrm{SI})$, even though the $\mathrm{N} 3-\mathrm{H} \cdots \mathrm{O} 4$ contacts connecting the $\mathrm{AB}_{\mathrm{i}}$ synthons along $\mathbf{b}$ are $\approx 0.1$ $\AA$ longer $\left(d_{\mathrm{H} \cdots \mathrm{O}}=1.93\right.$ and $1.91 \AA$ $v s .1 .84$ and $\left.1.82 \AA\right)$ in the optimized lattice. On the contrary, the cyclic HB pattern within the ASU is slightly reinforced, with $d_{\mathrm{H} \cdots \mathrm{O}}$ distances in the $\mathrm{N} 1-\mathrm{H} \cdots \mathrm{O} 1$ contacts lowering to 1.89 and $1.86 \AA$ in the relaxed structure compared to 1.90 and $1.89 \AA$ in the experimental one. These differences are, however, partly due to the increased cell edge lengths in the DFT-optimized lattice. Anyhow, the high stability of the non-centrosymmetric $P c a 2_{1}$ polymorph implies that a strong thermodynamic driving force towards the crystal form actually observed by means of the X-ray analysis exists.

Figure 9 shows the Hirshfeld surface fingerprint plots of the optimized solid-state centrosymmetric structures listed in Table 4 to be compared with those shown in Figure 3 for experimental PYRAC. The plots for the A and B conformers within the Pca2 $2_{1}$ form at the optimized DFT geometry are quite similar to their experimental counterparts (Figure S17, SI). 




(a)

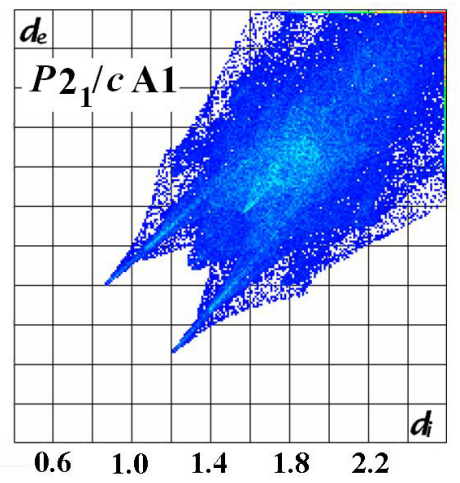

(b)

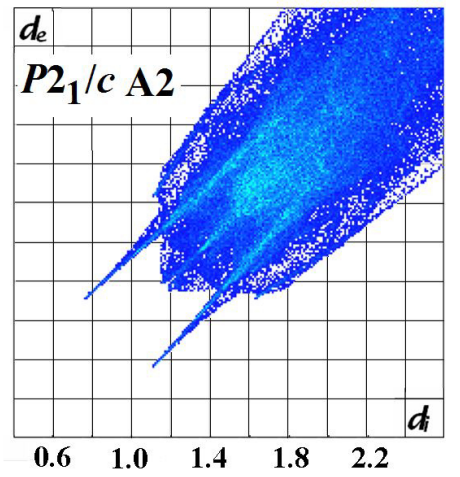

(c)

Figure 9. Colour online. Hirshfeld surface fingerprint plots (see Figure 4) for computer optimized centrosymmetric solid state structures of PYRAC at the B3LYP 6-311G(p,d) level of theory. (a) $P \overline{1}$, synthon $\mathrm{AA}_{\mathrm{i}} 1$; (b) $P 2_{1} / c$, synthon $\mathrm{AA}_{\mathrm{i}} 1$; (c) $P 2{ }_{1} / c$, synthon $\mathrm{AA}_{\mathrm{i}} 2$.

Less negative $E_{\text {latt }}$ in conjunction with lower packing coefficients and densities of the centrosymmetric structures are due to less stabilizing non-covalent patterns. From Figure 9 some familiar features are recognizable, such as the symmetric, parallel spikes due to the $\mathrm{N}-\mathrm{H} \cdots \mathrm{O}$ HBs. Note that these spikes are significantly longer for the $\mathrm{AA}_{\mathrm{i}} 2 P 2{ }_{1} / c$ structure, as it contains the strong cyclic $\mathrm{HB}$ arrangement between facing 5-membred rings lacking in the $\mathrm{AA}_{\mathrm{i}}$ 1-based structures. The less dense and efficient packing of conformers shown in Fig. 9 respect to experimental PYRAC is however detectable from the dramatic increment of $d_{\mathrm{i}} / d_{\mathrm{e}}$ contacts in the upper right corner of their fingerprint plots. A continuum of points at high $d_{\mathrm{i}}+d_{\mathrm{e}}$ implies that, on average, atoms very far from the surface are found with high frequency, i.e. the structure contains a significant amount of empty space. Actually, when the $\mathrm{AA}_{\mathrm{i}} 1$ building block is considered, all the HB donors and acceptors are involved in cyclic patterns within the pair itself (Figure 6): no other functions are available to set up extended $\mathrm{NH} \cdots \mathrm{O}$ HB chains, so the crystal is made up by 'isolated' clusters (see Figure S18, SI). Similarly, in the $\mathrm{AA}_{\mathrm{i}} 2$ building block the only available N3-H amide hydrogen has no accessible acceptors in its neighbourhoods. Moreover, both the $\mathrm{AA}_{\mathrm{i}} 1$ and 2 pairs have an overall dipole moment constrained to be zero by their $C_{\text {i }}$ point symmetry. Therefore, electrostatics plays a less important role than in $P c a 2_{1}$ and the dispersive-repulsive energy balance is expected to dominate crystal packing in simulated centrosymmetric polymorphs. Final structures likely result from the tendency of terminal hydrocarbon groups to alleviate steric hindrance. Such a scenario can be associated to an abundance of ancillary $\mathrm{H} \cdots \mathrm{H}$ contacts, as it has been found in linear hydrocarbons: ${ }^{39 a}$ accordingly, all the plots in Figure 9 visibly exhibit the peak along the main diagonal that is associated to this feature.

Analogue conclusions and on a more quantitative basis, can be drawn by comparing the molecule...molecule pair interaction energies at their solid-state geometries. Details on the 
quantum-mechanically optimized structures here investigated are reported and thoroughly discussed in Section S8, SI.

2728Errore. I-segnalibronon èdefinite.

\section{Conclusions.}

A thorough experimental and theoretical study of the crystal packing and interaction energetics was performed on PYRAC, a non-centrosymmetric polar racemate obtained as a synthesis intermediate during the production of novel antitrypanosomal drugs. The most relevant building block in the crystal is a heterochiral asymmetric $\left(C_{1}\right)$ molecular pair, whose individual components differ by their relative conformations (A and B) and are linked to each other by a strong cyclic HB pattern. In turn, each pair is connected to neighbouring building blocks by extended $\mathrm{NH} \cdots \mathrm{O}$ chains. Our main objective was to explore the reasons underlying the lack of inversion symmetry in this structure.

Two equivalent interconversion pseudorotatory paths between $\mathrm{A}$ and $\mathrm{B}$ conformers were found. They occur in two steps, are very fast at ordinary temperatures and involve other two stable structures, $\mathrm{M}$ and $\mathrm{G}$, not present in the crystalline compound. From the relative stability of the stationary points on the PES, it results that molecules spend most of their time $(\approx 53 \%)$ in the A conformation, while the $\mathrm{B}$ one is far less populated $(\approx 7 \%)$ and $\mathrm{G}, \mathrm{M}$ occur with similar frequency $(\approx 20 \%)$. Even though $\mathrm{A}, \mathrm{B}$ and $\mathrm{M}$ species all form stable adducts when interacting with their inversion-related analogues, $\mathrm{B}$ and $\mathrm{M}$ minima are too scarcely populated at ordinary temperatures to ensure a sufficiently frequent formation of the synthon to seed a crystal embryo. What ultimately determines the preference for the $C_{1} \mathrm{AB}_{\mathrm{i}}$ adduct in the crystal over the $\mathrm{AA}_{\mathrm{i}}$ ones is a strong thermodynamic driving force towards the $P c a 2_{1}$ structure. Actually, other centrosymmetric synthons invariably generate crystal forms with cohesive energies, densities and packing efficiencies significantly less favourable than those found experimentally. This denotes the inability of centrosymmetric structures to set up extended $\mathrm{NH} \cdots \mathrm{O}$ HB networks across the crystal lattice, as either all available $\mathrm{N}-\mathrm{H}$ donors are involved in intra-synthon HBs, or because no $\mathrm{HB}$ acceptors could come sufficiently close to amide free hydrogen atoms.

The present study implies some shortcomings. Our picture of the PYRAC energy landscape is limited, as we focused just on the analysis of a representative series of centrosymmetric polymorphs as possible competitors of the experimental structure. Therefore, we cannot a priori exclude that other non-centrosymmetric structures might exist, possibly able to compete with the $P c a 2_{1}$ one. Another limitation is due to the restricted sampling of the conformational space of isolated PYRAC, as different kinds of crystal nuclei might be produced by exploring further stationary structures. More complex scenarios are even possible: for example, interconversion paths 
could exist among centrosymmetric and non-centrosymmetric synthons $\left(\mathrm{XX}_{\mathrm{i}} \leftrightarrow \mathrm{XY}_{\mathrm{i}} \leftrightarrow \mathrm{YY}_{\mathrm{i}}\right.$, with $\mathrm{X}, \mathrm{Y}=\mathrm{A}, \mathrm{B}, \mathrm{G}, \mathrm{M}) .{ }^{52}$ Eventually, the solvent might have a role that remains to be explored.

On the other hand, our methodology relies on the knowledge of a spontaneously formed crystal structure and we would almost by sure unable to predict the correct crystal structure of PYRAC from scratch as the approach inherently lacks predictability. Nevertheless, the knowledge of accurate single-crystal X-ray structures and of their experimentally-derived charge densities might become increasingly important as a precious clue for improving the accuracy and reliability of the computational recipes for computer-modelling of crystalline materials.

\section{Acknowledgements}

The Italian supercomputing centre CINECA and Università degli Studi di Milano are gratefully acknowledged for computational time provided through the CINECA-UNIMI convention. Mr. Pietro Colombo (Italian CNR) is thanked for technical assistance. Dr. Silvia Rizzato (Unimi, Chemistry Dept.) is also acknowledged for providing lab facilities. Partial funding of the Center of Materials Crystallography (Aarhus, DK) by means of the Danish National Research Foundation was also appreciated.

Supporting Information Available. Discussion on the phase purity of PYRAC crystals. Details of the diffraction data treatment and least-squares procedures. Technical comnputational details. Temperature-dependent thermal effects. Symmetry breaking in the asymmetric unit. Computational LST procedure. Intermolecular $\mathrm{C}-\mathrm{H} \cdots \mathrm{A}, \mathrm{A}=\mathrm{O}, \mathrm{N}$ hydrogen bonded contacts. Modelling of solidstate molecule-molecule interaction energies. Energy decomposition for the multipolar charge density and CLP models. Energetics of computer generated polymorphs by means of the CLP model. Hirshfeld surface fingerprint plots for the $P c a 2_{1}$ bulk optimized structure. Comparison among the in-crystal molecular A, B conformers and structures found after in vacuo geometry optimization. Packing schemes for computer generated structures. Role of symmetry operators. Crystallographic information file. This information is available free of charge via the Internet at http://pubs.acs.org/ 


\section{References}

(1) Kurki-Suonio, K.; Isr. J. Chem. 1977, 16, 115-123.

(2) (a) Allieta, M.; Scavini, M.; Lo Presti, L.; Coduri, M.; Loconte, L.; Cappelli, S.; Oliva, C.; Ghigna, P.; Pattison, P.; Scagnoli, V. Phys. Rev. B, 2013, 88, 21414, 1-10; (b) Lo Presti, L.; Allieta, M.; Scavini, M.; Ghigna, P.; Loconte, L.; Scagnoli, V.; Brunelli, M. Phys. Rev. B 2011, 84, 104107, $1-9$.

(3) Curtin, D. Y. Chem. Rev. 1981, 81, 525

(4) Lang, S. B. Phys. Today 2005, 58, 31-36

(5) Nalwa, H. S.; Miyata, S. Nonlinear Optics of Organic Molecules and Polymers, CRC press Inc, Boca Raton (FL), 1997.

(6) (a) Centore, R.; Fusco, S.; Jazbinsek, M.; Capobianco, A.; Peluso, A. CrystEngComm 2013, 15, 3318-3325. (b) Centore, R.; Jazbinsek, M.; Tuzi, A.; Roviello, A.; Capobianco, A.; Peluso, A. CrystEngComm 2012, 14, 2645-2653.

(7) Desiraju, G. R.; Vittal, J. J.; Ramanan, V. Crystal Engineering: A Textbook. World Scientific Publishing Co. Pte. Ltd., Singapore, 2011.

(8) Macchi, P. Chimia 2014, 68, 31-37

(9) (a) Woodley, S. M.; Catlow, R. Nature Materials 2008, 7, 937-946; (b) Price, S. L. Chem. Soc. Rev. 2014, 43, 2098-2111.

(10) (a) Gavezzotti, A. Cryst. Res. Technol. 2013, 48, 793-810. (b) Gavezzotti, A. Molecular Aggregation: Structure Analysis and Molecular Simulation of Crystals and Liquids. Oxford University Press, 2007, Oxford, UK; (c) Gavezzotti, A. Acc. Chem. Res. 1994, 27, 309-314.

(11) (a) Threlfall, T. Org. Process Res. Dev. 2003, 7, 1017-1027; (b) Hedges, L. O.; Whitelam, S. J. Chem. Phys. 2011, 135, 164902, 1-7.

(12) (a) Desiraju, R. G. Nature Materials 2002, 1, 77-79; (b) Gavezzotti, A.; Filippini, G. Chem. Commun. 1998, 287-294. (c) Price, S. L. Acta Crystallogr., Sect. B 2013, 69, 313-328.

(13) Bardwell, D. A.; Adjiman, C. S.; Arnautova, Y. A.; Bartashevich, E.; Boerrigter, S. X. M.; Braun, D. E.; Cruz-Cabeza, A. J.; Day, G. M.; Della Valle, R. G.; Desiraju, G. R.; van Eijck, B. P.; Facelli, J. C.; Ferraro, M. B.; Grillo, D.; Habgood, M.; Hofmann, D. W. M.; Hofmann, F.; Jose, K. V. J.; Karamertzanis, P. G.; Kazantsev, A. V.; Kendrick, J.; Kuleshova, L. N.; Leusen, F. J. J.; Maleev, A. V.; Misquitta, A. J.; Mohamed, S.; Needs, R. J.; Neumann, M. A.; Nikylov, D.; Orendt, A. M.; Pal, R.; Pantelides, C. C.; Pickard, C. J.; Price, L. S.; Price, S. L.; Scheraga, H. A.; van de Streek, J.; Thakur, T. S.; Tiwari, S.; Venuti, E.; Zhitkov, I. K. Acta Crystallogr., Sect. B. 2011, 67, $535-551$.

(14) Lo Presti, L. ; Soave, R.; Longhi, M.; Ortoleva, E. Acta Crystallogr., Sect. B 2010, 66, 527543. 
(15) Destro, R.; Loconte, L.; Lo Presti, L.; Roversi, P.; Soave, R. Acta Crystallogr., Sect. A 2004, 60, 365-370.

(16) Tamborini, L.; Pinto, A.; Smith, T. K.; Major, L. L.; Iannuzzi, M. C.; Cosconati, S.; Marinelli, L.; Novellino, E.; Lo Presti, L.; Wong, P. E.; Barrett, M. P.; De Micheli, C.; Conti, P. ChemMedChem 2012, 7, 1623-1634.

(17) Groom, C. R.; Allen, F. H. Angew. Chem., Int. Ed. 2014, 53, 662-671.

(18) Allen, H. Acta Crystallogr., Sect. B 2002, 58, 380-388.

(19) Dalhus, B.; Görbitz, C. H. Acta Crystallogr., Sect. B 2000, 56, 715-719

(20) Kostyanovsky, R. G.; Kostyanovsky, V. R.; Kadorkina, G. K. Mendeleev Commun. 2009, 19, $17-18$.

(21) (a) D’Oria, E.; Karamertzanis, P. G.; Price, S. L. Cryst. Growth Des. 2010, 10, 1749-1756; (b) Gourlay, M. D.; Kendrick, J.; Leusen, F. J. J. Cryst. Growth Des. 2007, 7, 56-63; (c) Avalos, M.; Babiano, R.; Cintas, P.; Jimenez, J. L.; Palacios, J. C. Origins Life Evol. Biospheres 2004, 34: 391405

(22) (a) Destro, R.; Sartirana, E.; Loconte, L.; Soave, R.; Colombo, P.; Destro, C.; Lo Presti, L. Cryst. Growth Des. 2013, 13, 4571-4582; (b) Saleh, G.; Soave, R.; Lo Presti, L.; Destro, R. Chem. - Eur. J. 2013, 19, 3490 - 3503; (c) Lo Presti, L.; Ellern, A.; Destro, R.; Soave, R.; Lunelli, B. J. Phys. Chem. A 2011, 115, 12695-12707; (d) Lo Presti, L.; Ellern, A.; Destro, R.; Lunelli, B. J. Phys. Chem. A 2009, 113, 3186-3196; (e) Biliškov, N.; Kojic-Prodic, B.; Mali, G.; Molcanov, K.; Stare, J. J. Phys. Chem. A 2011, 115, 3154-3166. (f) Johnstone, R. D. L.; Lennie, A. R.; Parker, S. F.; Parsons, S.; Pidcock, E.; Richardson, P. R.; Warren, J. E.; Wood, P. A. CrystEngComm 2010, $12,1065-1078$.

(23) CCDC 1014249 contains the supplementary crystallographic data for this paper. These data can be obtained free of charge from the Cambridge Crystallographic Data Centre via www.ccdc.cam.ac.uk/data_request/cif.

(24) Bruker. SMART and SAINT. Bruker AXS Inc., 1999 Madison, Wisconsin, USA

(25) Bruker SADABS 2007, Bruker AXS Inc., Madison, Wisconsin, USA.

(26) Bruker XPREP - Reciprocal Space Exploration v.2005/2, 2005, Bruker AXS Inc., Madison, Wisconsin, USA.

(27) Hansen, N. K.; Coppens, P. Acta Crystallogr., Sect. A 1978, 34, 909-921.

(28) Volkov, A.; Macchi, P.; Farrugia, L. J.; Gatti, C.; Mallinson, P.; Richter, T.; Koritsanszky, T. XD2006-A Computer Program Package for Multipole Refinement, Topological Analysis of Charge Densities and Evaluation of Intermolecular Energies from Experimental and Theoretical Structure Factors, 2006. See also http://xd.chem.buffalo.edu/. 
(29) (a) Bader, R. F. W. Atoms in Molecules: A Quantum Theory; Oxford University Press: Oxford, U.K., 1990. (b) Matta, C. F.; Boyd, R. J. The Quantum Theory of Atoms in Molecules: From Solid State to DNA and Drug Design; Wiley-VCH Verlag GmbH \& Co. KGaA: Weinheim, 2007, (c) Gatti, C.; Macchi, P. Modern Charge-Density Analysis; Dordrecht: Springer, 2012

(30) (a) Dovesi, R.; Saunders, V. R.; Roetti, C.; Orlando, R.; Zicovich-Wilson, C. M.; Pascale, F.; Civalleri, B.: Doll, K.; Harrison, N. M.; Bush, I. J.; D’Arco P.; Llunell, M. CRYSTAL09 User's Manual (University of Torino, Torino, 2009); (c) Dovesi, R.; Orlando, R.; Civalleri, B.; Roetti, C.; Saunders, V. R.; Zicovich-Wilson C. M. Z. Kristallogr. 2005, 220, 571

(31) (a) Gatti, C.; Saunders, V.R.; Roetti, C. J. Chem. Phys. 1994, 101, 10686-10696; (b) Spackman, M.A.; Mitchell, A.S. Phys. Chem. Chem. Phys. 2001, 3, 1518-1523

(32) (a) Becke, A.D. J. Chem. Phys. 1993, 98, 5648-5652; (b) Lee, C.; Yang, W.; Parr, R.G. Phys. Rev. B 1988, 37, 785-789; (c) Vosko, S. H.; Wilk, L.; Nusair, M. Can. J. Phys. 1980, 58, 12001211; (d) Stephens, P.J.; Devlin, F.J.; Chabalowski, C.F.; Frisch, M.J. J. Phys. Chem. 1994, 98, 11623-11627.

(33) (a) Gavezzotti, A. The Coulomb - London - Pauli (CLP) model of intermolecular interaction Description and user's manual v. 3.6.2013, 2013, Milano. (b) Gavezzotti, A. New J. Chem. 2011, 35, 1360-1368. (c) Dunitz, J. D.; Gavezzotti, A. Angew. Chem., Int. Ed. 2005, 44, 1766-1787 (d) Dunitz, J. D.; Gavezzotti, A. Chem. Soc. Rev. 2009, 38, 2622-2633.

(34) Krishnan, R.; Binkley, J.S.; Seeger, R.; Pople, J.A. J. Chem. Phys. 1980, 72, 650-654.

(35)Frisch, M. J.; Trucks, G. W.; Schlegel, H. B.; Scuseria, G. E.; Robb, M. A.; Cheeseman, J. R.; Scalmani, G.; Barone, V. et al. Gaussian 09, Revision A.02; Gaussian, Inc.: Wallingford, CT, 2009. (36) Boys, S. F.; Bernardi, F. Molecular Physics 1970, 19, 553-566.

(37) Bertolasi, V.; Gilli, P.; Ferretti, V.; Gilli, G. Acta Crystallogr., Sect.B 2001, 57, 591-598.

(38) Lo Presti, L.; Soave, R.; Destro, R. J. Phys. Chem. B 2006, 110, 6405-6414.

(39) (a) McKinnon, J. J.; Mitchell, A. S.; Spackman, M. A. Chem. - Eur. J. 1998, 4, 2136-2141; (b) Spackman, M. A.; McKinnon, J. K. Cryst. Growth Des. 2002, 66, 378-392.

(40) The promolecular density is a theoretical reference model density. It is defined as the charge density distribution due to non-interacting, spherical and neutral atoms placed at the same positions they have in the molecule. The corresponding sum over the crystal is called procrystal density. A Hirshfeld surface encloses the region of space where the procrystal density is dominated by the promolecular contribution of a given molecule.

(41) (a) Rohl, A. L.; Moret, M.; Kaminsky, W.; Claborn, K.; McKinnon, J. J.; Kahr, B. Cryst. Growth Des. 2008, 8, 4517-4525. (b) Braun, D. E.; Többens, D. M.; Kahlenberg, V.; Ludescher, J.; Griesser, U. J. Cryst. Growth Des. 2008, 8, 4109-4119. 
(42) Wolff, S. K.; Grimwood, D. J.; McKinnon, J. J.; Jayatilaka, D.; Spackman, M. A. 2007. CrystalExplorer 2.1. University of Western Australia. See also http://www.hirshfeldsurface.net.

(43) Cremer, D.; Pople, J.A. J.Am. Chem. Soc. 1975, 97, 1354-1358

(44) For example, the torsion angle $\tau_{\mathrm{C} 12-\mathrm{C} 11-\mathrm{C} 10-\mathrm{O} 5}$ amounts to $134.70(6) \mathrm{deg}$ for the A molecule and 42.0(2) deg for its homochiral B analogue.

(45) The kinetic definition of supramolecular synthons as given by Desiraju, R. G. J. Chim. Sci. 2010, 122, 667-675, namely 'structural units that express the core features of a crystal structure and which encapsulate the essence of crystals in terms of molecular recognition', is here intended. This definition relies on a probabilistic model, 'concerned with the frequency of occurrence of substructural units which are representative not only of the final crystal but also of patterns that evolve during crystallization'. As the $\mathrm{AB}_{\mathrm{i}}$ and $\mathrm{AA}_{\mathrm{i}}$ adducts favourably score against most of the centrosymmetric $\mathrm{XX}_{\mathrm{i}}$ adducts (see the discussion below), they can be considered as effective synthons for stable and close-packed structures.

(46) Such rescaling procedure somewhat privileges thermodynamics at the expense of kinetics, i.e. it assumes an equilibrium population of interacting conformers. When the equilibrium is broken, for example when crystallization starts, the relative ranking of different pairs could vary.

(47) This model implicitly takes into account the energy contribution due to internal relaxation, but still neglects steric (reorientation) effects. The latter are reasonably much less important than the total interaction energy of the pair.and are also expected to be essentially constant throughout the adducts here considered, as the interacting species are chemically the same.

(48) (a) Saleh, G.; Gatti, C.; Lo Presti, L.; Contreras-Garcia, Chem. - Eur. J. 2012, 18, 15523-15536

(b) Saleh, G.; Lo Presti, L.; Gatti, C.; Ceresoli, D. J. Appl. Crystallogr. 2013, 46, 1513-1517

(49) Dobson, J. F.; McLennan, K.; Rubio, A.; Wang, J.; Gould, T.; Lee, H. M.; Dinte, B. P. Aust. J. Chem. 2001, 54, 513-527

(50) (a) Chisholm, J. A.; Motherwell, S.; Tulip, P. R.; Parsons, S.; Clark, S. J. Crystal Growth \& Design, 2005, 5, 1437-1442; (b) Montanari B.; Ballone, P.; Jones, R. O. J. Chem. Phys. 1998, 108, 6947-6951.

(51) In particular, estimates for Ecoh cannot be strictly related to thermodynamic quantities such as the sublimation enthalpies at $0 \mathrm{~K}, \Delta_{\text {sub }} \mathrm{H}^{0}(0)$, because they also lack the in-crystal vibrational zeropoint correction.

(52) We believe, however, that $X_{\mathrm{i}} \leftrightarrow \mathrm{XY}_{\mathrm{i}}$ interconversion paths should be less important than direct $\mathrm{X}-\mathrm{X}_{\mathrm{i}}$ or $\mathrm{X}-\mathrm{Y}_{\mathrm{i}}$ recognition events in solution, as conformational changes of individual molecules within the synthon are expected to be somewhat slowed by steric hindrance. 


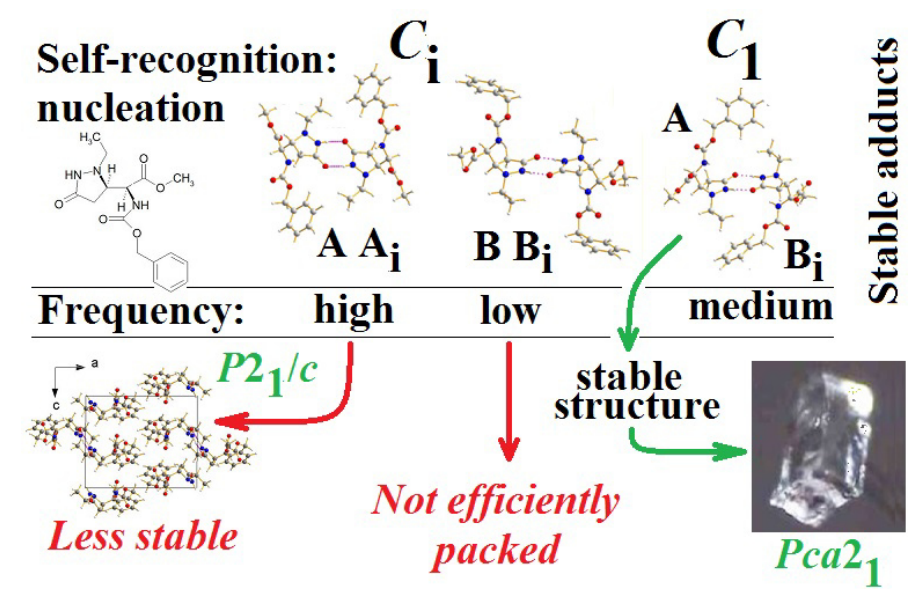

TABLE OF CONTENT GRAPHIC: PYRAC can form many stable gas-phase adducts, both centrosymmetric and non-centrosymmetric. While the former lead to less stable crystal structures, a strong thermodynamic bias forces the $C_{1}$ adduct to yield an acentric polar Pca2 $2_{1}$ crystal. 